\title{
Synthesis of Phosphinopeptides via the Mannich Ligation
}

\author{
Bonan Li, Shuzong Cai, Da-Ming Du, and Jiaxi Xu* \\ Beijing National Laboratory for Molecular Sciences (BNLMS), Key Laboratory of Bioorganic Chemistry \\ and Molecular Engineering of Ministry of Education, College of Chemistry and Molecular Engineering, \\ Peking University, Beijing 100871, People's Republic of China \\ jxxu@pku.edu.cn
}

\section{Supplemental Materials}

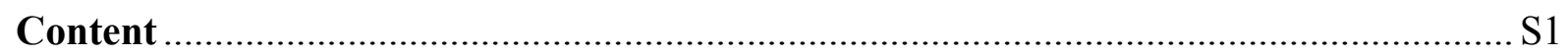

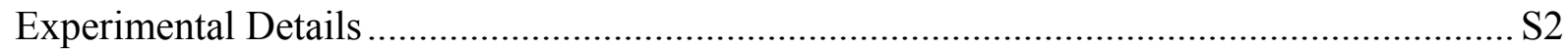

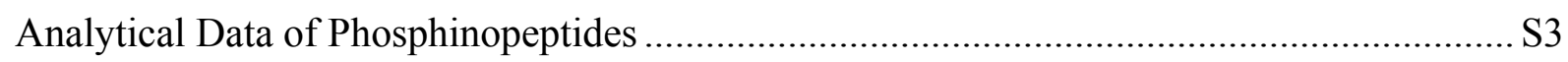

Copies of ${ }^{1} \mathrm{H}$ and ${ }^{13} \mathrm{C}$ NMR Spectra of Phosphinopeptides ............................................... S9 


\section{Experimental Details}

General Method. Amino acid and peptide esters were obtained via neutralization of their hydrochlorides with ammonia and extraction with ethyl acetate. Aryldichlorophosphines were prepared according to literature procedure ${ }^{1}$ and their analytical data are identical to reported ones. ${ }^{1,2}$ Acetonitrile was refluxed with calcium hydride and freshly distilled prior to use. All reactions were performed under a nitrogen atmosphere. ${ }^{1} \mathrm{H},{ }^{13} \mathrm{C}$ and ${ }^{31} \mathrm{P}$ NMR spectra were recorded at $300 \mathrm{MHz}$ in $\mathrm{CDCl}_{3}$ with TMS as an internal standard and $85 \% \mathrm{H}_{3} \mathrm{PO}_{4}$ as an external standard for ${ }^{31} \mathrm{P}$ NMR. IR spectra were determined directly. MS spectra were obtained on an ESI mass spectrometer.

General Procedure: To a solution of Cbz-amino acid/peptide amide $(3 \mathrm{mmol})$ and aldehyde $(3.5 \mathrm{mmol})$ in dried acetonitrile $(10 \mathrm{~mL})$ was added arylphosphine dichloride $(3.5 \mathrm{mmol})$ under a nitrogen atmosphere. The resulting solution was stirred at room temperature for $12 \mathrm{~h}$. After addition of diisopropylethylamine $(1.29 \mathrm{~g}, 10 \mathrm{mmol})$, the reaction mixture was stirred for $10 \mathrm{~min}$. Amino acid/peptide ester $(6 \mathrm{mmol})$ in dried acetonitrile $(5 \mathrm{~mL})$ was added. The reaction mixture was stirred for additional $24 \mathrm{~h}$. After removal of solvent, the residue was dissolved in ethyl acetate $(250 \mathrm{~mL})$. The solution was washed with saturated sodium bicarbonate $(100 \mathrm{~mL} \times 5)$, saturated brine $(100 \mathrm{~mL} \times 2), 0.1 \mathrm{~mol} / \mathrm{L}$ citric acid $(0.02 \mathrm{~mol} / \mathrm{L}$ citric acid for non-glycine esters) $(100 \mathrm{~mL} \times 5)$, saturated brine $(100 \mathrm{~mL} \times 2)$, dried over anhydrous sodium sulfate. After concentration at reduced pressure, the residue was recrystallized in a mixture of ethyl acetate and diethyl ether to afford colorless powder crystals.

\section{References}

1. Buchner, B.; Lockhart, H. J. Am. Chem. Soc. 1951, 73, 755.

2. Weinberg, K. G. J. Org. Chem. 1975, 40, 3586. 


\section{Analytical Data of Phosphinopeptides}

Phenyl[ $N$-[ $N$-benzyloxycarbonylglycinyl]-1-aminophenylmethyl]phosphinic acid (Scheme 1)<smiles>O=C(CNC(=O)OCc1ccccc1)NC(c1ccccc1)P(=O)(O)c1ccccc1</smiles>

White solid, m.p. $312-315{ }^{\circ} \mathrm{C}$; IR $v\left(\mathrm{~cm}^{-1}\right): 1702(\mathrm{C}=\mathrm{O}), 1119(\mathrm{P}=\mathrm{O}) ;{ }^{1} \mathrm{H}$ NMR $(300 \mathrm{MHz}$, $\left.\mathrm{CDCl}_{3}\right) \delta: 4.07-4.17(\mathrm{~m}, 3 \mathrm{H}), 5.00(\mathrm{~s}, 2 \mathrm{H}), 5.13(\mathrm{~d}, J=11.7 \mathrm{~Hz}, 1 \mathrm{H}), 6.00(\mathrm{~d}, J=25.2 \mathrm{~Hz}, 1 \mathrm{H})$, 6.98-7.34 (m, 15H), $7.79(\mathrm{~d}, J=26.1 \mathrm{~Hz}, 1 \mathrm{H}) ;{ }^{13} \mathrm{C} \mathrm{NMR}\left(75.5 \mathrm{MHz}, \mathrm{CDCl}_{3}\right) \delta: 47.8\left(\mathrm{~d}, J_{\mathrm{P}-\mathrm{C}}=17.3\right.$ Hz), 67.4, 71.9, 126.5, 127.8, 128.0, 128.3, 128.5, 128.7, 129.3, 135.7, 138.6, 139.1, 153.3, 170.6 $\left(\mathrm{d}, J_{\mathrm{P}-\mathrm{C}}=17.9 \mathrm{~Hz}\right) ;{ }^{31} \mathrm{P}$ NMR $\left(121.5 \mathrm{MHz}, \mathrm{CDCl}_{3}\right): \delta 34.9$. HRMS (ESI) Calcd. for $\mathrm{C}_{23} \mathrm{H}_{23} \mathrm{~N}_{2} \mathrm{O}_{5} \mathrm{P}$ $\left(\mathrm{M}+\mathrm{H}^{+}\right)$439.1423; Found 439.1421.

Ethyl $N$-[phenyl[ $N$-[ $N$-benzyloxycarbonylglycinyl]-1-aminophenylmethyl]phosphinyl]glycine (Table 2, entry 1)<smiles>CCOC(=O)CNP(=O)(c1ccccc1)C(NC(=O)CNC(=O)OCc1ccccc1)c1ccccc1</smiles>

White solid, m.p. 179.0-180.5 ${ }^{\circ} \mathrm{C}$; IR $v\left(\mathrm{~cm}^{-1}\right)$ : $1724(\mathrm{C}=\mathrm{O}), 1743(\mathrm{C}=\mathrm{O}), 1673(\mathrm{C}=\mathrm{O}), 1237$ $(\mathrm{P}=\mathrm{O}) ;{ }^{1} \mathrm{H} \mathrm{NMR}\left(300 \mathrm{MHz}, \mathrm{CDCl}_{3}\right) \delta: 1.15(\mathrm{t}, J=7.2 \mathrm{~Hz}, 3 \mathrm{H}), 3.29(\mathrm{~d}, J=6.3 \mathrm{~Hz}, 2 \mathrm{H}), 3.52(\mathrm{~d}, J$ $=6.3 \mathrm{~Hz}, 1 \mathrm{H}), 3.72-3.98(\mathrm{~m}, 2 \mathrm{H}), 4.06(\mathrm{q}, J=7.2 \mathrm{~Hz}, 2 \mathrm{H}), 5.00(\mathrm{~s}, 2 \mathrm{H}), 5.80(\mathrm{t}, J=10.5 \mathrm{~Hz}, 1 \mathrm{H})$, $6.04(\mathrm{~s}, 1 \mathrm{H}), 7.28-7.62(\mathrm{~m}, 15 \mathrm{H}), 9.09(\mathrm{~d}, J=8.1 \mathrm{~Hz}, 1 \mathrm{H}) ;{ }^{13} \mathrm{C} \mathrm{NMR}\left(75.5 \mathrm{MHz}, \mathrm{CDCl}_{3}\right) \delta: 13.9$, 41.4, 44.0, $53.1\left(\mathrm{~d}, J_{\mathrm{P}-\mathrm{C}}=99.3 \mathrm{~Hz}\right), 61.4,66.6,127.5,127.9,128.0,128.3,128.7,128.8,129.2$, 132.1, 132.3, 132.8, 134.7, 136.3, 156.1, 169.0, 170.8; ${ }^{31} \mathrm{P} \mathrm{NMR}\left(121.5 \mathrm{MHz}, \mathrm{CDCl}_{3}\right) \delta: 30.6$. HRMS (ESI) Calcd. for $\mathrm{C}_{27} \mathrm{H}_{30} \mathrm{~N}_{3} \mathrm{O}_{6} \mathrm{P}\left(\mathrm{M}+\mathrm{H}^{+}\right)$524.1950; Found 524.1948.

Ethyl $N$-[ 4-methylphenyl[ $N$-[ $N$-benzyloxycarbonylglycinyl]-1-aminophenylmethyl]phosphinyl]glycine (Table 2 , entry 2 )<smiles>CCOC(=O)CNP(=O)(c1ccc(C)cc1)C(NC(=O)CNC(=O)OCc1ccccc1)c1ccccc1</smiles> 
White solid, m.p. 151-153 ${ }^{\circ} \mathrm{C}$; IR $v\left(\mathrm{~cm}^{-1}\right): 1715(\mathrm{C}=\mathrm{O}), 1675(\mathrm{C}=\mathrm{O}), 1182(\mathrm{P}=\mathrm{O}) ;{ }^{1} \mathrm{H}$ NMR $\left(300 \mathrm{MHz}, \mathrm{CDCl}_{3}\right) \delta: 1.14(\mathrm{t}, J=6.9 \mathrm{~Hz}, 3 \mathrm{H}), 2.41(\mathrm{~s}, 3 \mathrm{H}) ; 3.18-3.33(\mathrm{~m}, 3 \mathrm{H}) ; 3.72-3.89(\mathrm{~m}, 2 \mathrm{H})$; 4.04 (q, $J=7.2 \mathrm{~Hz}, 2 \mathrm{H}), 4.98$ (s, 2H), 5.71 (t, $J=9.9 \mathrm{~Hz}, 1 \mathrm{H}), 5.93$ (s, br, 1H), 7.27-7.75 (m, 14H), $8.95(\mathrm{~s}, \mathrm{br}, 1 \mathrm{H}) ;{ }^{13} \mathrm{C} \mathrm{NMR}\left(75.5 \mathrm{MHz}, \mathrm{CDCl}_{3}\right) \delta: 13.9,21.7,41.4,44.0,53.0\left(\mathrm{~d}, J_{\mathrm{P}-\mathrm{C}}=99.3 \mathrm{~Hz}\right)$, $61.3,66.5,126.5,127.8,128.3,128.7,128.8,129.1,129.5,129.6,132.2,134.8,136.3,143.4$, 156.0, 168.8, 170.7; ${ }^{31} \mathrm{P}$ NMR (121.5 MHz, $\mathrm{CDCl}_{3}$ ) $\delta$ : 30.7. HRMS (ESI) Calcd. for $\mathrm{C}_{28} \mathrm{H}_{32} \mathrm{~N}_{3} \mathrm{O}_{6} \mathrm{P}$ $\left(\mathrm{M}+\mathrm{H}^{+}\right)$538.2107; Found 538.2109.

\section{Ethyl [ $N$-[4-chlorophenyl[ $N$-[ $N$-benzyloxycarbonylglycinyl]-1-aminophenylmethyl]Phosp}

hinyl]glycine (Table 2, entry 3 )<smiles>CCOC(=O)CNP(=O)(c1ccc(Cl)cc1)C(NC(=O)CNC(=O)OCc1ccccc1)c1ccccc1</smiles>

White solid, m.p. $152-155^{\circ} \mathrm{C}$; IR $v\left(\mathrm{~cm}^{-1}\right)$ : $1717(\mathrm{C}=\mathrm{O}), 1673(\mathrm{C}=\mathrm{O}), 1212(\mathrm{P}=\mathrm{O}) ;{ }^{1} \mathrm{H}$ NMR $\left(300 \mathrm{MHz}, \mathrm{CDCl}_{3}\right) \delta: 1.13(\mathrm{t}, J=7.2 \mathrm{~Hz}, 3 \mathrm{H}), 3.26(\mathrm{~m}, 2 \mathrm{H}), 3.67(\mathrm{~m}, 1 \mathrm{H}), 3.77-3.91(\mathrm{~m}, 2 \mathrm{H})$, $4.03(\mathrm{q}, J=6.9 \mathrm{~Hz}, 2 \mathrm{H}), 5.00(\mathrm{~s}, 2 \mathrm{H}), 5.81(\mathrm{t}, J=10.8 \mathrm{~Hz}, 1 \mathrm{H}), 6.00(\mathrm{~s}, \mathrm{br}, 1 \mathrm{H}), 7.27-7.78(\mathrm{~m}$, $14 \mathrm{H}), 8.97(\mathrm{~d}, J=7.8 \mathrm{~Hz}, 1 \mathrm{H}) ;{ }^{13} \mathrm{C} \mathrm{NMR}\left(75.5 \mathrm{MHz}, \mathrm{CDCl}_{3}\right) \delta: 13.9,41.2,43.9,52.8\left(\mathrm{~d}, J_{\mathrm{P}-\mathrm{C}}=\right.$ $100.5 \mathrm{~Hz}), 61.5,66.6,126.5,127.9,128.3,128.6,128.7,129.0,129.1,133.5,133.7,134.3,136.3$, 139.3, 156.2, 169.2, 170.7; ${ }^{31} \mathrm{P}$ NMR $\left(121.5 \mathrm{MHz}, \mathrm{CDCl}_{3}\right)$ 8: 30.4. HRMS (ESI) Calcd. for $\mathrm{C}_{27} \mathrm{H}_{29} \mathrm{ClN}_{3} \mathrm{O}_{6} \mathrm{P}\left(\mathrm{M}+\mathrm{H}^{+}\right)$558.1561; Found 558.1555.

Ethyl $N$-[phenyl[ $N$-[ $N$-benzyloxycarbonylglycinyl]-1-amino(4-methylphenyl)methyl]phosphinyllglycine (Table 2, entry 4)<smiles>CCOC(=O)CNP(=O)(c1ccccc1)C(NC(=O)CNC(=O)OCc1ccccc1)c1ccc(C)cc1</smiles>

White solid, m.p. 159.0-161.0 ${ }^{\circ} \mathrm{C}$; IR $v\left(\mathrm{~cm}^{-1}\right): 1746(\mathrm{C}=\mathrm{O}), 1724(\mathrm{C}=\mathrm{O}), 1675(\mathrm{C}=\mathrm{O}), 1239$ $(\mathrm{P}=\mathrm{O}) ;{ }^{1} \mathrm{H}$ NMR $\left(300 \mathrm{MHz}, \mathrm{CDCl}_{3}\right) \delta: 1.14(\mathrm{t}, J=7.2 \mathrm{~Hz}, 3 \mathrm{H}), 2.34(\mathrm{~s}, 3 \mathrm{H}), 3.29(\mathrm{t}, J=6.9 \mathrm{~Hz}$, $2 \mathrm{H}), 3.40-3.50(\mathrm{~m}, 1 \mathrm{H}), 3.70-3.94(\mathrm{~m}, 2 \mathrm{H}), 4.04(\mathrm{q}, J=7.2 \mathrm{~Hz} 2 \mathrm{H}), 4.95(\mathrm{~d}, J=11.7 \mathrm{~Hz}, 1 \mathrm{H}$ in $\left.\mathrm{CH}_{2}\right), 5.00\left(\mathrm{~d}, J=11.7 \mathrm{~Hz}, 1 \mathrm{H}\right.$ in $\left.\mathrm{CH}_{2}\right), 5.72(\mathrm{t}, J=10.5 \mathrm{~Hz}, 1 \mathrm{H}), 6.06(\mathrm{~d}, J=4.5 \mathrm{~Hz}, 1 \mathrm{H})$; 7.09-7.91 (m, 14H), $9.01(\mathrm{~d}, J=2.4 \mathrm{~Hz}, 1 \mathrm{H}) ;{ }^{13} \mathrm{C} \mathrm{NMR}\left(75.5 \mathrm{MHz}, \mathrm{CDCl}_{3}\right)$ 8: 13.9, 21.1, 41.4, 44.0, $52.7\left(\mathrm{~d}, J_{\mathrm{P}-\mathrm{C}}=100.0 \mathrm{~Hz}\right), 61.3,66.5,127.8,128.3,128.6,128.7,129.3,129.5,131.6,132.2$, 132.3, 132.6, 136.3, 138.1, 156.0, $168.8\left(\mathrm{~d}, J_{\mathrm{P}-\mathrm{C}}=7.1 \mathrm{~Hz}\right), 170.7 ;{ }^{31} \mathrm{P} \mathrm{NMR}\left(121.5 \mathrm{MHz}, \mathrm{CDCl}_{3}\right) \delta$ : 30.3. HRMS (ESI) Calcd. for $\mathrm{C}_{28} \mathrm{H}_{32} \mathrm{~N}_{3} \mathrm{O}_{6} \mathrm{P}\left(\mathrm{M}+\mathrm{H}^{+}\right)$538.2107; Found 538.2110. 
Ethyl $N$-[phenyl[ $N$-[ $N$-benzyloxycarbonylglycinyl]-1-amino(4-chlorophenyl)methyl]phosphinyl]glycine (Table 2, entry 5)<smiles>CCOC(=O)CNP(=O)(c1ccccc1)C(NC(=O)CNC(=O)OCc1ccccc1)c1ccc(Cl)cc1</smiles>

White solid, m.p. 176.0-177.0 ${ }^{\circ} \mathrm{C}$; IR $v\left(\mathrm{~cm}^{-1}\right)$ : 1737(C=O), $1721(\mathrm{C}=\mathrm{O}), 1677(\mathrm{C}=\mathrm{O}), 1180$ $(\mathrm{P}=\mathrm{O}) ;{ }^{1} \mathrm{H}$ NMR $\left(300 \mathrm{MHz}, \mathrm{CDCl}_{3}\right) \delta: 1.14(\mathrm{t}, J=7.2 \mathrm{~Hz}, 3 \mathrm{H}), 3.31(\mathrm{~d}, J=6.0 \mathrm{~Hz}, 2 \mathrm{H}), 3.69-3.95$ (m, 3H), $4.04(\mathrm{q}, J=6.9 \mathrm{~Hz}, 2 \mathrm{H}), 4.99(\mathrm{~s}, 2 \mathrm{H}), 5.80(\mathrm{t}, J=10.5 \mathrm{~Hz}, 1 \mathrm{H}), 6.06(\mathrm{~s}, 1 \mathrm{H}), 7.20-7.86$ $(\mathrm{m}, 14 \mathrm{H}), 9.08(\mathrm{~d}, J=7.5 \mathrm{~Hz}, 1 \mathrm{H}) ;{ }^{13} \mathrm{C} \mathrm{NMR}\left(75.5 \mathrm{MHz}, \mathrm{CDCl}_{3}\right) \delta: 13.9,41.5,44.0,52.4\left(\mathrm{~d}, J_{\mathrm{P}-\mathrm{C}}\right.$ $=99.9 \mathrm{~Hz}), 61.5,66.6,127.6,128.0,128.4,128.7,130.0,130.1,131.9,132.0,132.8,133.3,134.2$, 136.3, 156.2, 169.1, 170.7; ${ }^{31} \mathrm{P}$ NMR $\left(121.5 \mathrm{MHz}, \mathrm{CDCl}_{3}\right)$ $\delta: 31.2$. HRMS (ESI) Calcd. for $\mathrm{C}_{27} \mathrm{H}_{29} \mathrm{ClN}_{3} \mathrm{O}_{6} \mathrm{P}\left(\mathrm{M}+\mathrm{H}^{+}\right)$558.1561; Found 558.1554.

Ethyl $N$-[phenyl[ $N$-[ $N$-benzyloxycarbonylglycinyl]-1-amino-2-methylpropyl]phosphinyllglycine (Table 2 , entry 6 )<smiles>CCOC(=O)CNP(O)(c1ccccc1)(C(C)C)C(NC(=O)CNC(=O)OCc1ccccc1)c1ccccc1</smiles>

White solid, m.p. 160.0-162.0 ${ }^{\circ} \mathrm{C}$; IR $v\left(\mathrm{~cm}^{-1}\right)$ : $1732(\mathrm{C}=\mathrm{O}), 1662(\mathrm{C}=\mathrm{O}), 1203(\mathrm{P}=\mathrm{O}) ;{ }^{1} \mathrm{H}$ NMR $\left(300 \mathrm{MHz}, \mathrm{CDCl}_{3}\right) \delta: 0.85(\mathrm{~d}, J=6.6 \mathrm{~Hz}, 3 \mathrm{H}), 0.96(\mathrm{~d}, J=6.3 \mathrm{~Hz}, 3 \mathrm{H}), 1.24(\mathrm{t}, J=7.0 \mathrm{~Hz}, 3 \mathrm{H})$, $2.37(\mathrm{~m}, 1 \mathrm{H}), 3.42-3.53(\mathrm{~m}, 1 \mathrm{H}), 3.61-3.94(\mathrm{~m}, 3 \mathrm{H}), 3.94-4.04(\mathrm{~m}, 1 \mathrm{H}), 4.14(\mathrm{q}, J=7.0 \mathrm{~Hz}, 2 \mathrm{H})$, $4.47-4.51(\mathrm{~m}, 1 \mathrm{H}), 5.08(\mathrm{~s}, 2 \mathrm{H}), 5.93(\mathrm{~s}, \mathrm{br}, 1 \mathrm{H}), 7.16(\mathrm{~d}, J=9.9 \mathrm{~Hz}, 1 \mathrm{H}), 7.29-7.81(\mathrm{~m}, 10 \mathrm{H}) ;{ }^{13} \mathrm{C}$ NMR $\left(75.5 \mathrm{MHz}, \mathrm{CDCl}_{3}\right) \delta: 14.0,17.9,21.2\left(\mathrm{~d}, J_{\mathrm{P}-\mathrm{C}}=10.8 \mathrm{~Hz}\right), 28.0,41.4,44.4,52.9\left(\mathrm{~d}, J_{\mathrm{P}-\mathrm{C}}=\right.$ $101.7 \mathrm{~Hz}), 61.4,67.0,127.9,128.1,128.4,128.6,130.0,132.4,136.1,156.5,169.7,170.9 ;{ }^{31} \mathrm{P}$

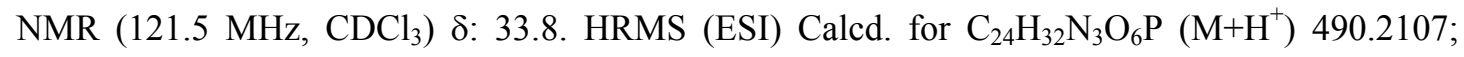
Found 490.2113.

Ethyl $N$-[phenyl[ $N$-[ $N$-benzyloxycarbonylglycinyl]-1-aminobutyl]phosphinyl]glycine (Table 2 , entry 7 )<smiles>CCCC(NC(=O)CNC(=O)OCc1ccccc1)P(=O)(NCC(=O)OCC)c1ccccc1</smiles>

White solid, m.p. 126.0-130.0 ${ }^{\circ} \mathrm{C}$; IR $v\left(\mathrm{~cm}^{-1}\right)$ : $1737(\mathrm{C}=\mathrm{O}), 1677(\mathrm{C}=\mathrm{O}), 1240(\mathrm{P}=\mathrm{O}) ;{ }^{1} \mathrm{H}$ NMR $\left(300 \mathrm{MHz}, \mathrm{CDCl}_{3}\right) \delta: 0.76(\mathrm{t}, J=6.6 \mathrm{~Hz}, 3 \mathrm{H}), 1.13(\mathrm{t}, J=7.2 \mathrm{~Hz}, 3 \mathrm{H}), 1.34(\mathrm{~m}, 2 \mathrm{H}), 1.79(\mathrm{~m}, 1 \mathrm{H})$, 
$3.40(\mathrm{~m}, 1 \mathrm{H}), 3.55(\mathrm{~m}, 1 \mathrm{H}), 3.69-3.85(\mathrm{~m}, 2 \mathrm{H}), 4.05(\mathrm{q}, J=7.2 \mathrm{~Hz}, 2 \mathrm{H}), 4.18(\mathrm{~m}, 1 \mathrm{H}), 4.42(\mathrm{~m}$, $1 \mathrm{H}), 4.96(\mathrm{~s}, 2 \mathrm{H}), 6.02(\mathrm{~s}, 1 \mathrm{H}), 7.23-7.44(\mathrm{~m}, 10 \mathrm{H}), 7.68(\mathrm{~d}, J=10.5 \mathrm{~Hz}, 1 \mathrm{H}), 7.71(\mathrm{~d}, J=8.1 \mathrm{~Hz}$, $1 \mathrm{H}) ;{ }^{13} \mathrm{C}$ NMR $\left(75.5 \mathrm{MHz}, \mathrm{CDCl}_{3}\right) \delta: 13.4,14.0,19.2\left(\mathrm{~d}, J_{\mathrm{P}-\mathrm{C}}=12.5 \mathrm{~Hz}\right), 29.7,41.4,44.3,48.2(\mathrm{~d}$, $\left.J_{\mathrm{P}-\mathrm{C}}=101.3 \mathrm{~Hz}\right), 61.3,66.8,127.8,128.0,128.4,128.5,132.3,132.5,132.7,136.1,156.4,169.7$, 171.0; ${ }^{31} \mathrm{P}$ NMR (121.5 MHz, $\left.\mathrm{CDCl}_{3}\right)$ 8: 35.3. HRMS (ESI) Calcd. for $\mathrm{C}_{24} \mathrm{H}_{32} \mathrm{~N}_{3} \mathrm{O}_{6} \mathrm{P}\left(\mathrm{M}+\mathrm{H}^{+}\right)$ 490.2107; Found 490.2112.

\section{Ethyl $N$-[phenyl[ $N$-[ $N$-benzyloxycarbonyl-D/L-alaninyl]-1-aminophenylmethyl]phos-} phinyl]glycine (Table 2 , entry 8 )<smiles>CCOC(=O)CNP(=O)(c1ccccc1)C(NC(=O)C(C)NC(=O)OCc1ccccc1)c1ccccc1</smiles>

White solid, m.p. 180.0-182.0 ${ }^{\circ} \mathrm{C}$; IR $v\left(\mathrm{~cm}^{-1}\right)$ : $1732(\mathrm{C}=\mathrm{O}), 1717(\mathrm{C}=\mathrm{O}), 1657(\mathrm{C}=\mathrm{O}), 1194$ $(\mathrm{P}=\mathrm{O}) ;{ }^{1} \mathrm{H}$ NMR $\left(300 \mathrm{MHz}, \mathrm{CDCl}_{3}\right) \delta: 0.97(\mathrm{~d}, J=7.0 \mathrm{~Hz}, 3 \mathrm{H}), 1.14(\mathrm{t}, J=6.9 \mathrm{~Hz}, 3 \mathrm{H}), 3.29(\mathrm{~m}$, 2H), $3.50(\mathrm{~m}, 1 \mathrm{H}), 4.05(\mathrm{q}, J=7.0 \mathrm{~Hz}, 2 \mathrm{H}), 4.49(\mathrm{~m}, 1 \mathrm{H}), 4.93\left(\mathrm{~d}, J=12.0 \mathrm{~Hz}, 1 \mathrm{H}\right.$ in $\left.\mathrm{CH}_{2}\right), 5.00$ (d, $J=12.0 \mathrm{~Hz}, 1 \mathrm{H}$ in $\left.\mathrm{CH}_{2}\right), 5.73(\mathrm{t}, J=10.8 \mathrm{~Hz}, 1 \mathrm{H}), 5.90(\mathrm{~d}, J=8.4 \mathrm{~Hz}, 1 \mathrm{H}), 7.25-7.92(\mathrm{~m}$, $15 \mathrm{H}), 8.81(\mathrm{~d}, J=6.6 \mathrm{~Hz}, 1 \mathrm{H}) ;{ }^{13} \mathrm{C}$ NMR $\left(75.5 \mathrm{MHz}, \mathrm{CDCl}_{3}\right) \delta: 14.0,19.5,41.3,50.1,52.9(\mathrm{~d}$, $\left.J_{\mathrm{P}-\mathrm{C}}=96.9 \mathrm{~Hz}\right), 61.4,66.5,127.9,128.3,128.6,128.7,128.8,132.1,132.2,132.6,134.6,136.4$, 155.5, 170.8, 172.4; ${ }^{31} \mathrm{P}$ NMR (121.5 MHz, $\mathrm{CDCl}_{3}$ ) $\delta$ : 30.4. HRMS (ESI) Calcd. for $\mathrm{C}_{28} \mathrm{H}_{32} \mathrm{~N}_{3} \mathrm{O}_{6} \mathrm{P}$ $\left(\mathrm{M}+\mathrm{H}^{+}\right)$538.2107; Found 538.2101.

Ethyl $N$-[phenyl[ $N$-[ $N$-benzyloxycarbonyl- $\beta$-alaninyl]-1-aminophenylmethyl]phosphinyl]glycine (Table 2, entry 9)<smiles>CCOC(=O)CNP(=O)(c1ccccc1)C(NC(=O)CCNC(=O)OCc1ccccc1)c1ccccc1</smiles>

White solid, m.p. 160.0-162.0 ${ }^{\circ} \mathrm{C}$; IR $v\left(\mathrm{~cm}^{-1}\right)$ : $1747(\mathrm{C}=\mathrm{O}), 1702(\mathrm{C}=\mathrm{O}), 1662(\mathrm{C}=\mathrm{O}), 1236$ $(\mathrm{P}=\mathrm{O}) ;{ }^{1} \mathrm{H}$ NMR $\left(300 \mathrm{MHz}, \mathrm{CDCl}_{3}\right) \delta: 1.15(\mathrm{t}, J=7.0 \mathrm{~Hz}, 3 \mathrm{H}), 2.33(\mathrm{~m}, 2 \mathrm{H}), 3.26-3.82(\mathrm{~m}, 5 \mathrm{H})$, $4.06(\mathrm{~d}, J=7.0 \mathrm{~Hz}, 2 \mathrm{H}), 5.00(\mathrm{~s}, 2 \mathrm{H}), 5.59(\mathrm{~s}, 1 \mathrm{H}), 5.72(\mathrm{t}, J=9.9 \mathrm{~Hz}, 1 \mathrm{H}), 7.28-7.88(\mathrm{~m}, 15 \mathrm{H})$, $8.39(\mathrm{~d}, J=7.5 \mathrm{~Hz}, 1 \mathrm{H}) ;{ }^{13} \mathrm{C} \mathrm{NMR}\left(75.5 \mathrm{MHz}, \mathrm{CDCl}_{3}\right) \delta: 14.0,35.4,37.3,41.4,53.1\left(\mathrm{~d}, J_{\mathrm{P}-\mathrm{C}}=\right.$ $99.3 \mathrm{~Hz}), 61.6,66.3,127.9,128.3,128.5,128.6,128.7,128.9,132.2,132.3,132.8,134.5,136.6$, 156.2, 170.8, 171.4; ${ }^{31} \mathrm{P}$ NMR (121.5 MHz, $\mathrm{CDCl}_{3}$ ) $8: 30.7$. HRMS (ESI) Calcd. for $\mathrm{C}_{28} \mathrm{H}_{32} \mathrm{~N}_{3} \mathrm{O}_{6} \mathrm{P}$ $\left(\mathrm{M}+\mathrm{H}^{+}\right)$538.2107; Found 538.2112. 
Methyl $N$-[phenyl[ $N$-[ $N$-benzyloxycarbonylglycinyl]-1-aminophenylmethyl]phosphinyl]-Lphenylalanine (Table 2, entry 10)<smiles>COC(=O)C(Cc1ccccc1)NP(=O)(c1ccccc1)[C@@H](NC(=O)CNC(=O)OCc1ccccc1)c1ccccc1</smiles><smiles>COC(=O)C(Cc1ccccc1)NP(=O)(O)c1ccccc1C(NC(=O)CNC(=O)OCc1ccccc1)c1ccccc1</smiles>

White solid, m.p.179.0-182.0 ${ }^{\circ} \mathrm{C}$; IR $v\left(\mathrm{~cm}^{-1}\right): 1721(\mathrm{C}=\mathrm{O}), 1672(\mathrm{C}=\mathrm{O}), 1192(\mathrm{P}=\mathrm{O}) ;{ }^{1} \mathrm{H}$ NMR (300 MHz, $\left.\mathrm{CDCl}_{3}\right)$ ): 2.64-2.84 (m, 2H), 3.48 (s, 3H), 3.63-3.76 (m, 2H), 3.79-3.86 (m, 2H), 4.99 $(\mathrm{s}, 2 \mathrm{H}), 5.60 \& 5.79(\mathrm{t}, J=15.9 \mathrm{~Hz} 1 \mathrm{H}), 5.75(\mathrm{~s}, \mathrm{br}, 1 \mathrm{H}), 6.67-7.88(\mathrm{~m}, 20 \mathrm{H}), 8.84 \& 8.99(\mathrm{~d}, J=$ $6.0 \mathrm{~Hz}, 1 \mathrm{H}) ;{ }^{13} \mathrm{C}$ NMR $\left(75.5 \mathrm{MHz}, \mathrm{CDCl}_{3}\right) \delta: 40.6,44.0,51.9,52.5\left(\mathrm{~d}, J_{\mathrm{P}-\mathrm{C}}=102.4 \mathrm{~Hz}\right), 54.2$, 66.6, 126.5, 126.7, 126.9, 127.9, 128.3, 128.6, 128.9, 129.2, 129.4, 132.0, 132.1, 132.3, 132.5, 134.6, 135.7, 136.4, 156.1, 168.9, 172.9; ${ }^{31} \mathrm{P}$ NMR (121.5 MHz, $\left.\mathrm{CDCl}_{3}\right): \delta 30.2: 29.7$ (1.07:1.00) . HRMS (ESI) Calcd. for $\mathrm{C}_{33} \mathrm{H}_{34} \mathrm{~N}_{3} \mathrm{O}_{6} \mathrm{P}\left(\mathrm{M}+\mathrm{H}^{+}\right) 600.2263$; Found 600.2271 .

Ethyl $N$-[phenyl[ $N$-[ $N$-benzyloxycarbonylglycinyl]-1-aminophenylmethyl]phosphinyl]$\beta$-alanine (Table 2, entry 12)<smiles>CCOC(=O)CCNP(=O)(c1ccccc1)C(NC(=O)CNC(=O)OCc1ccccc1)c1ccccc1</smiles>

White solid, m.p. 165.0-167.0 ${ }^{\circ} \mathrm{C}$; IR $v\left(\mathrm{~cm}^{-1}\right)$ : $1723(\mathrm{C}=\mathrm{O}), 1672(\mathrm{C}=\mathrm{O}), 1178(\mathrm{P}=\mathrm{O}) ;{ }^{1} \mathrm{H}$ NMR $\left(300 \mathrm{MHz}, \mathrm{CDCl}_{3}\right) \delta: 1.15(\mathrm{t}, J=7.2 \mathrm{~Hz}, 3 \mathrm{H}), 2.19(\mathrm{t}, J=6.0 \mathrm{~Hz}, 2 \mathrm{H}), 2.81(\mathrm{~m}, 2 \mathrm{H}), 3.41(\mathrm{~m}, 1 \mathrm{H})$, 3.67-3.97 (m, 2H), $4.01(\mathrm{q}, J=7.2 \mathrm{~Hz}, 2 \mathrm{H}), 4.98(\mathrm{~s}, 2 \mathrm{H}), 5.65(\mathrm{t}, J=10.5 \mathrm{~Hz}, 1 \mathrm{H}), 5.95(\mathrm{~s}, 1 \mathrm{H})$, 7.25-7.91 (m, 15H), $9.01(\mathrm{~d}, J=6.3 \mathrm{~Hz}, 1 \mathrm{H}) ;{ }^{13} \mathrm{C}$ NMR (75.5 MHz, $\left.\mathrm{CDCl}_{3}\right): \delta$ 14.0, 35.9, 36.0, $44.0,53.0\left(\mathrm{~d}, J_{\mathrm{P}-\mathrm{C}}=100.0 \mathrm{~Hz}\right), 60.5,66.6,127.9,128.3,128.6,128.7,128.8,129.7,132.2,132.3$, 132.6, 134.9, 136.3, $156.0168 .8,172.0 ;{ }^{31} \mathrm{P}$ NMR (121.5 MHz, $\left.\mathrm{CDCl}_{3}\right): \delta 30.8$. HRMS (ESI) Calcd. for $\mathrm{C}_{28} \mathrm{H}_{32} \mathrm{~N}_{3} \mathrm{O}_{6} \mathrm{P}\left(\mathrm{M}+\mathrm{H}^{+}\right)$538.2107; Found 538.2098.

Ethyl $N$-[ $N$-[phenyl[ $N$-[ $N$-benzyloxycarbonylglycinyl]-1-aminophenylmethyl]phosphinyl]glycinyl]glycine (Table 2, entry 13)<smiles>CCOC(=O)CNC(=O)CNP(=O)(c1ccccc1)C(NC(=O)CNC(=O)OCc1ccccc1)c1ccccc1</smiles>

White solid, m.p. $165.0-167.0{ }^{\circ} \mathrm{C}$; IR $v\left(\mathrm{~cm}^{-1}\right): 1717(\mathrm{C}=\mathrm{O}), 1699(\mathrm{C}=\mathrm{O}), 1655(\mathrm{C}=\mathrm{O}), 1181$ 
$(\mathrm{P}=\mathrm{O}) ;{ }^{1} \mathrm{H}$ NMR (300 MHz, $\left.\mathrm{CDCl}_{3}\right): \delta 1.20(\mathrm{t}, J=7.0 \mathrm{~Hz}, 3 \mathrm{H}), 3.21(\mathrm{~m}, 1 \mathrm{H}), 3.37(\mathrm{~m}, 1 \mathrm{H})$, $3.70-3.80(\mathrm{~m}, 3 \mathrm{H}), 3.82-4.00(\mathrm{~m}, 2 \mathrm{H}), 4.10(\mathrm{q}, J=7.0 \mathrm{~Hz}, 2 \mathrm{H}), 4.98(\mathrm{~s}, 2 \mathrm{H}), 5.79(\mathrm{t}, J=7.5 \mathrm{~Hz}$, $1 \mathrm{H}), 5.87(\mathrm{~s}, 1 \mathrm{H}), 7.14-7.62(\mathrm{~m}, 15 \mathrm{H}), 7.82(\mathrm{~d}, J=7.8 \mathrm{~Hz}, 1 \mathrm{H}), 7.86(\mathrm{~d}, J=7.5 \mathrm{~Hz}, 1 \mathrm{H}) ;{ }^{13} \mathrm{C}$ NMR (75.5 MHz, $\left.\mathrm{CDCl}_{3}\right): \delta 14.0,41.1,42.8,44.0,53.3\left(\mathrm{~d}, J_{\mathrm{P}-\mathrm{C}}=97.5 \mathrm{~Hz}\right), 61.3,66.7,126.6$, 127.8, 128.0, 128.4, 128.7, 128.8, 131.8, 131.9, 132.8, 134.6, 156.2, 168.7, 169.6, 170.2; ${ }^{31} \mathrm{P}$ NMR (121.5 MHz, $\left.\mathrm{CDCl}_{3}\right): \delta$ 32.4. HRMS (ESI) Calcd. for $\mathrm{C}_{29} \mathrm{H}_{33} \mathrm{~N}_{4} \mathrm{O}_{7} \mathrm{P}\left(\mathrm{M}+\mathrm{H}^{+}\right)$581.2165; Found 581.2162 .

Ethyl $N$-[phenyl[ $N$-[ $N$-[N-benzyloxycarbonylglycinyl]glycinyl]-1-aminophenylmethyl]phosphinyl]alanine (Table 2, entry 15)<smiles>CCOC(=O)CNP(=O)(c1ccccc1)C(NC(=O)CNC(=O)CNC(=O)OCc1ccccc1)c1ccccc1</smiles>

White solid, m.p. 159.0-163.0 ${ }^{\circ} \mathrm{C}$; IR $v\left(\mathrm{~cm}^{-1}\right)$ : $1737(\mathrm{C}=\mathrm{O}), 1673(\mathrm{C}=\mathrm{O}), 1241(\mathrm{P}=\mathrm{O}) ;{ }^{1} \mathrm{H}$ NMR $\left(300 \mathrm{MHz}, \mathrm{CDCl}_{3}\right) \delta: 1.14(\mathrm{t}, J=7.0 \mathrm{~Hz}, 3 \mathrm{H}), 3.34(\mathrm{~m}, 2 \mathrm{H}), 3.85-4.22(\mathrm{~m}, 5 \mathrm{H}), 4.08(\mathrm{q}, J=7.0$ $\mathrm{Hz}, 2 \mathrm{H}), 5.08(\mathrm{~s}, 2 \mathrm{H}), 5.64(\mathrm{t}, J=10.5 \mathrm{~Hz}, 1 \mathrm{H}), 6.17(\mathrm{~s}, \mathrm{br}, 1 \mathrm{H}), 7.12-7.71(\mathrm{~m}, 15 \mathrm{H}), 8.71(\mathrm{~s}, \mathrm{br}$, $2 \mathrm{H}) ;{ }^{13} \mathrm{C}$ NMR $\left(75.5 \mathrm{MHz}, \mathrm{CDCl}_{3}\right) \delta: 13.9,41.3,42.7,44.1,58.3\left(\mathrm{~d}, J_{\mathrm{P}-\mathrm{C}}=98.1 \mathrm{~Hz}\right), 61.3,66.7$, $127.9128 .1,128.3,128.5,128.7,132.1,132.5,134.6,136.5,156.7,168.7,169.6,171.0 ;{ }^{31} \mathrm{P} \mathrm{NMR}$ (121.5 MHz, $\left.\mathrm{CDCl}_{3}\right)$ ס: 31.6. HRMS (ESI) Calcd. for $\mathrm{C}_{29} \mathrm{H}_{33} \mathrm{~N}_{4} \mathrm{O}_{7} \mathrm{P}\left(\mathrm{M}+\mathrm{H}^{+}\right)$581.2165; Found 581.2172 . 
Copies of ${ }^{1} \mathrm{H}$ and ${ }^{13} \mathrm{C}$ NMR Spectra of Phosphinopeptides<smiles>O=C(CNC(=O)OCc1ccccc1)NC(c1ccccc1)P(=O)(O)c1ccccc1</smiles>

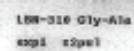

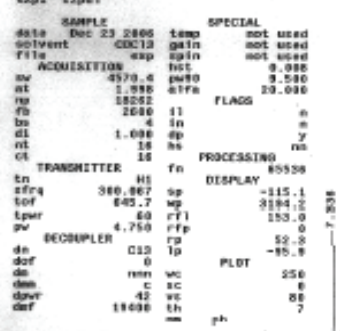

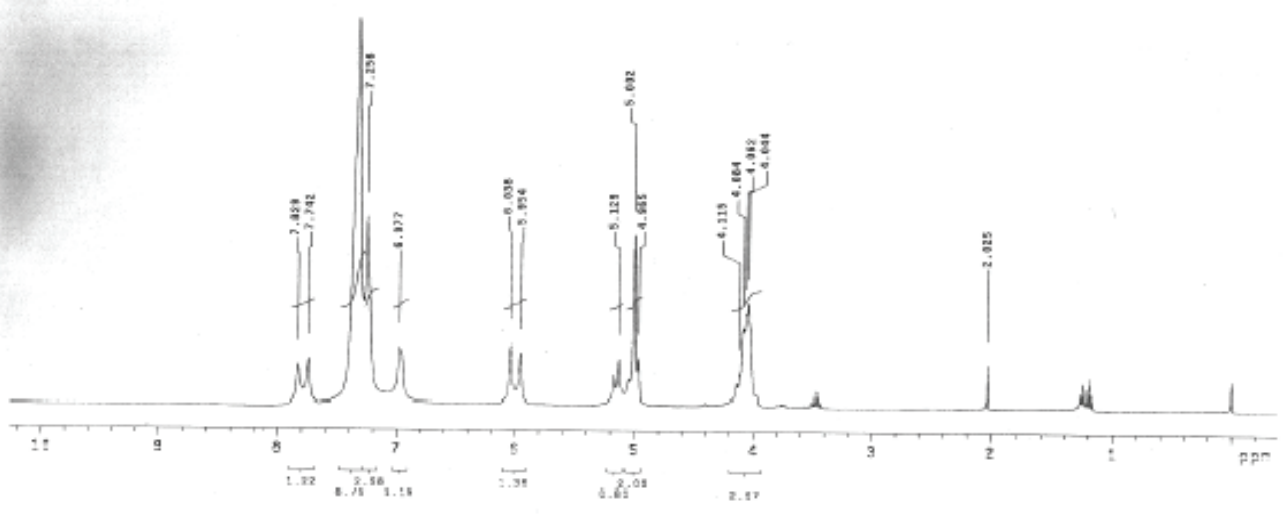

LEW-310 BIy-A19

$\operatorname{axp}$ sta13c
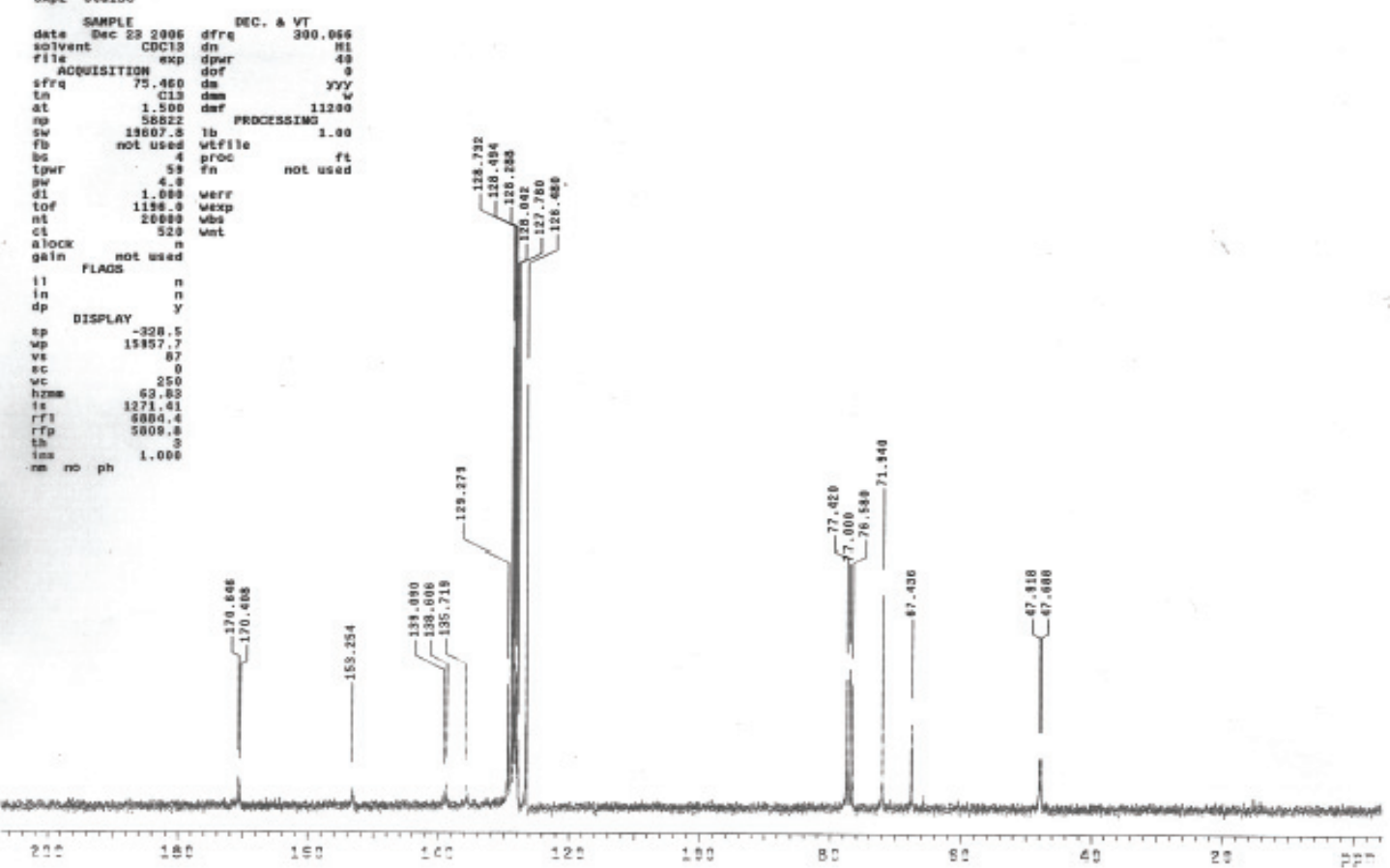
<smiles>CCOC(=O)CNP(=O)(c1ccccc1)C(NC(=O)CNC(=O)OCc1ccccc1)c1ccccc1</smiles>

Table 2, entry $1\left(300 \mathrm{MHz}, \mathrm{CDCl}_{3}\right)$

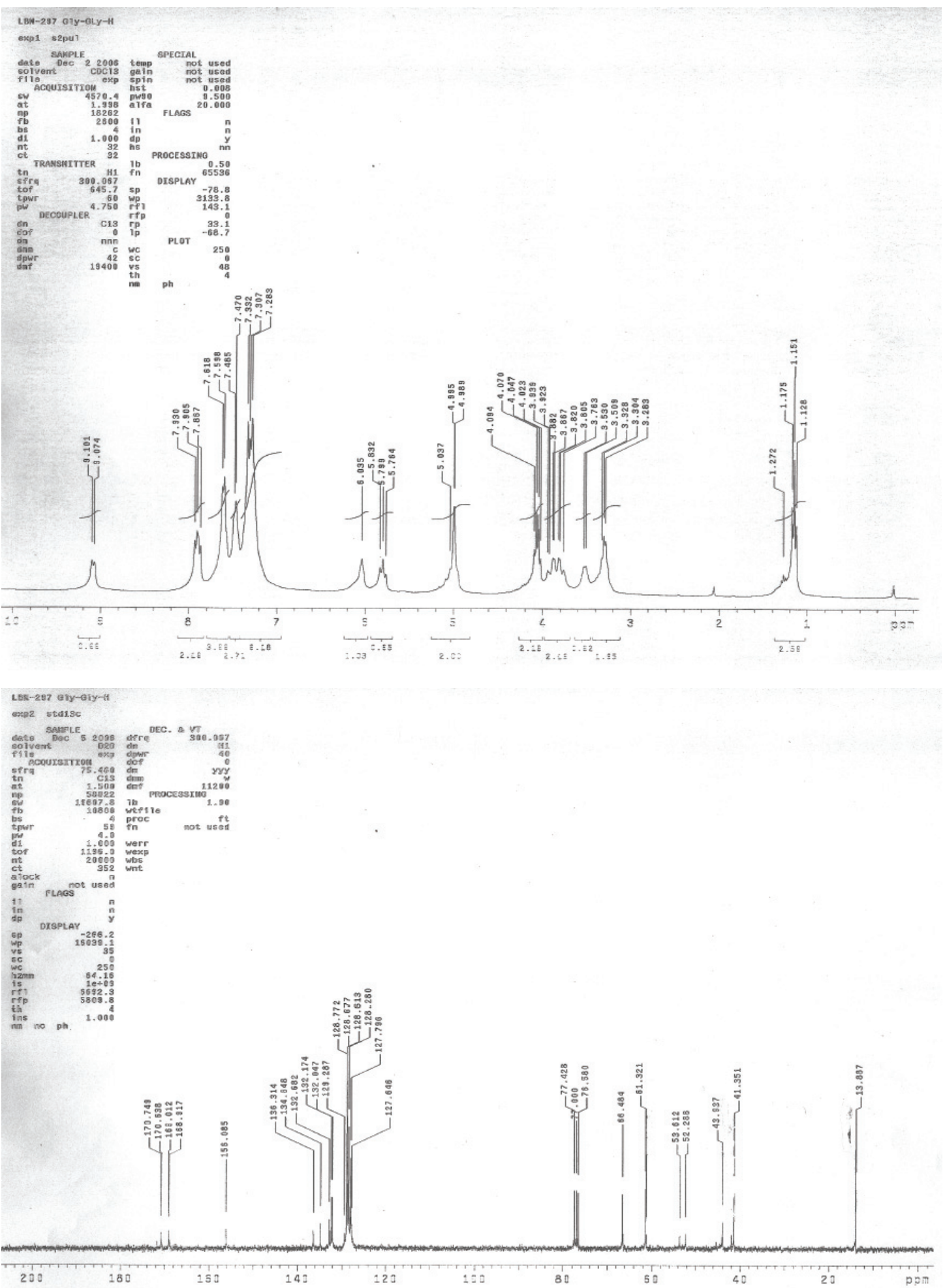


<smiles>CCOC(=O)CNP(=O)(c1ccc(C)cc1)C(NC(=O)CNC(=O)OCc1ccccc1)c1ccccc1</smiles>

Table 2 , entry $2\left(300 \mathrm{MHz}, \mathrm{CDCl}_{3}\right)$

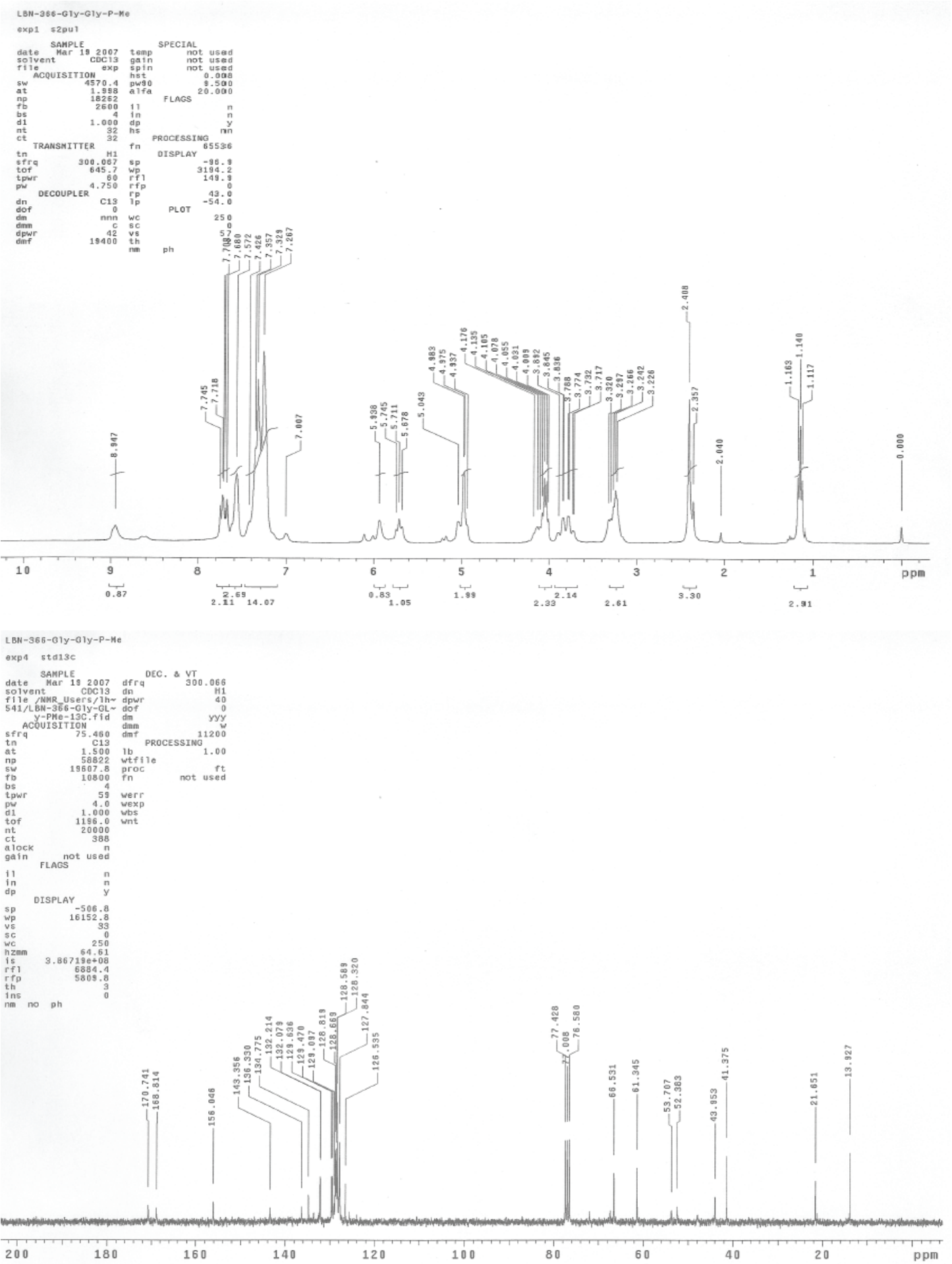


<smiles>CCOC(=O)CNP(=O)(c1ccc(Cl)cc1)C(NC(=O)CNC(=O)OCc1ccccc1)c1ccccc1</smiles>

Table 2, entry $3\left(300 \mathrm{MHz}, \mathrm{CDCl}_{3}\right)$

LBN-368 O1Y-GLY-PC1

exp1 52pui

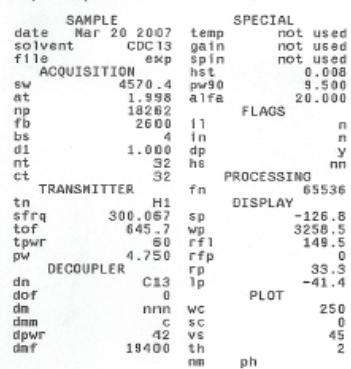

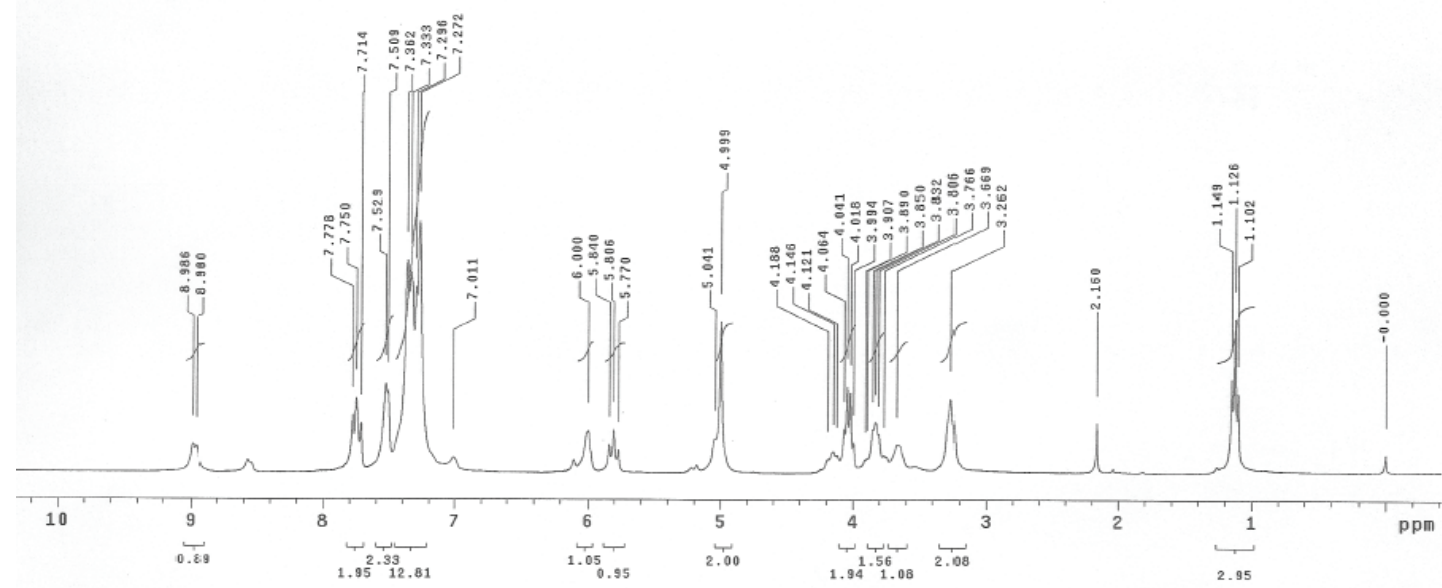

LEN-36B GIY-G1Y-PC)

exp2 sta13c

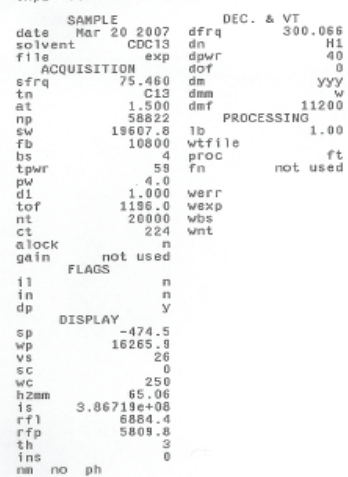

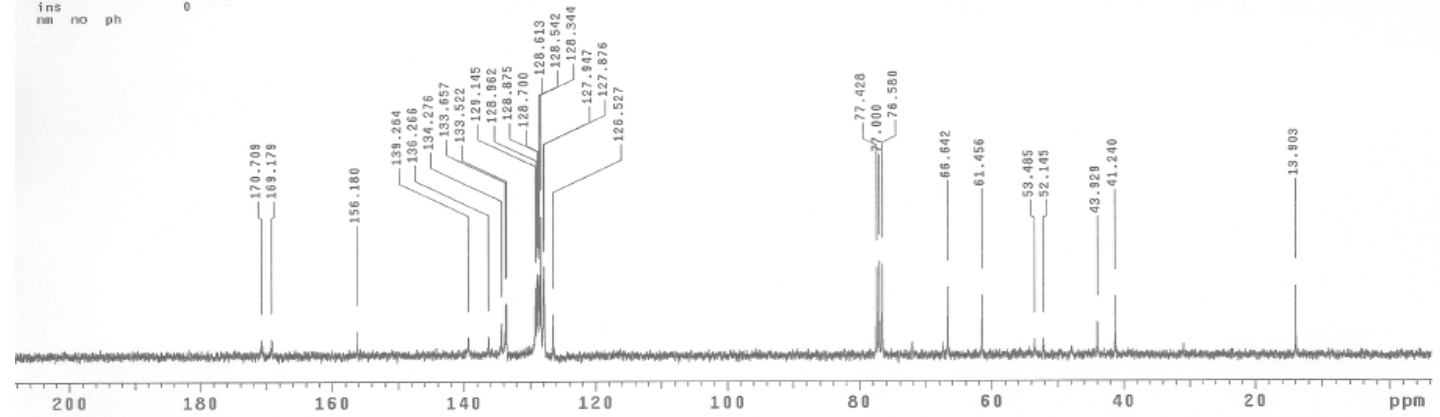


<smiles>CCOC(=O)CNP(=O)(c1ccccc1)C(NC(=O)CNC(=O)OCc1ccccc1)c1ccc(C)cc1</smiles>

Table 2, entry $4\left(300 \mathrm{MHz}, \mathrm{CDCl}_{3}\right)$
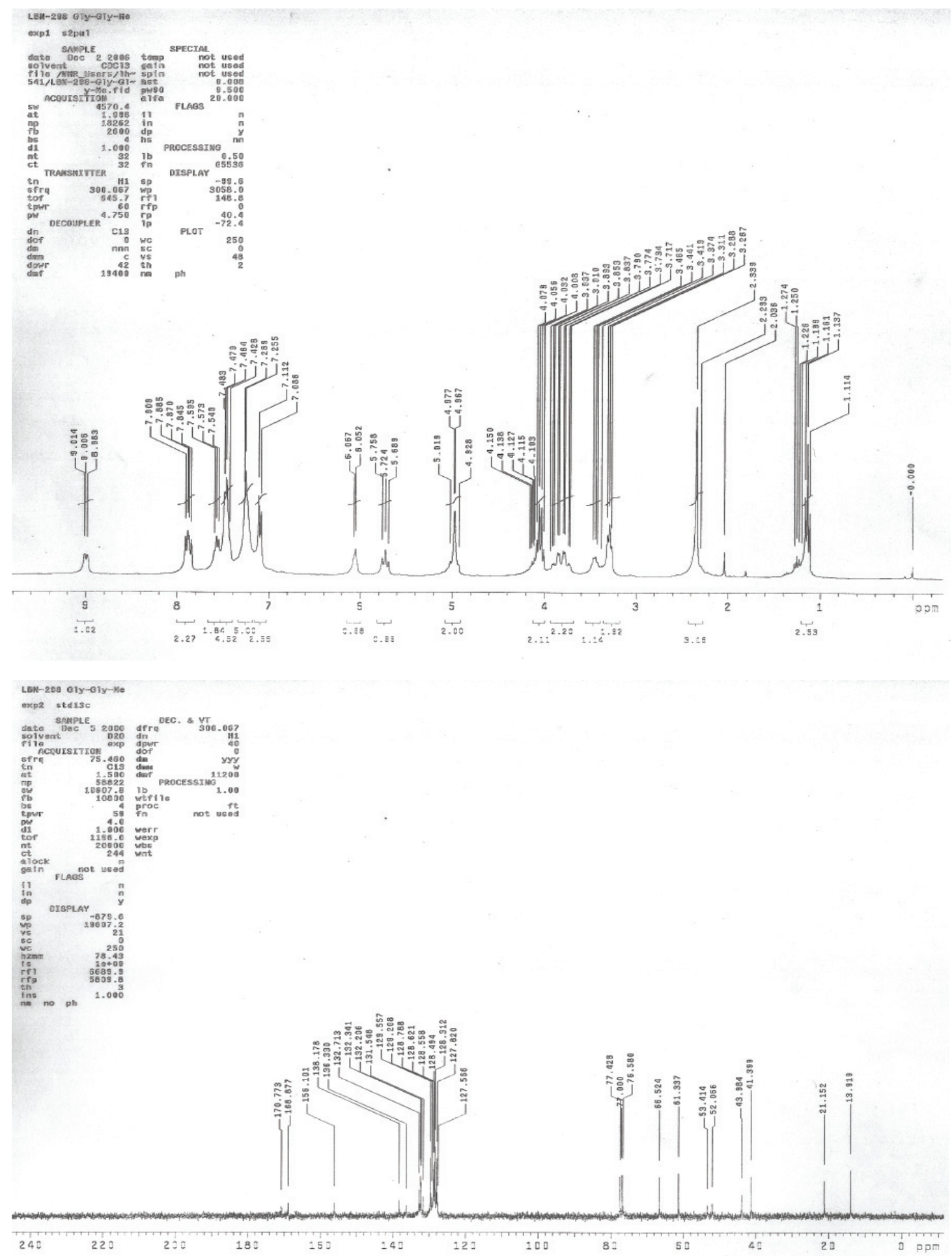
<smiles>CCOC(=O)CNP(=O)(c1ccccc1)C(NC(=O)CNC(=O)OCc1ccccc1)c1ccc(Cl)cc1</smiles>

Table 2 , entry $5\left(300 \mathrm{MHz}, \mathrm{CDCl}_{3}\right)$

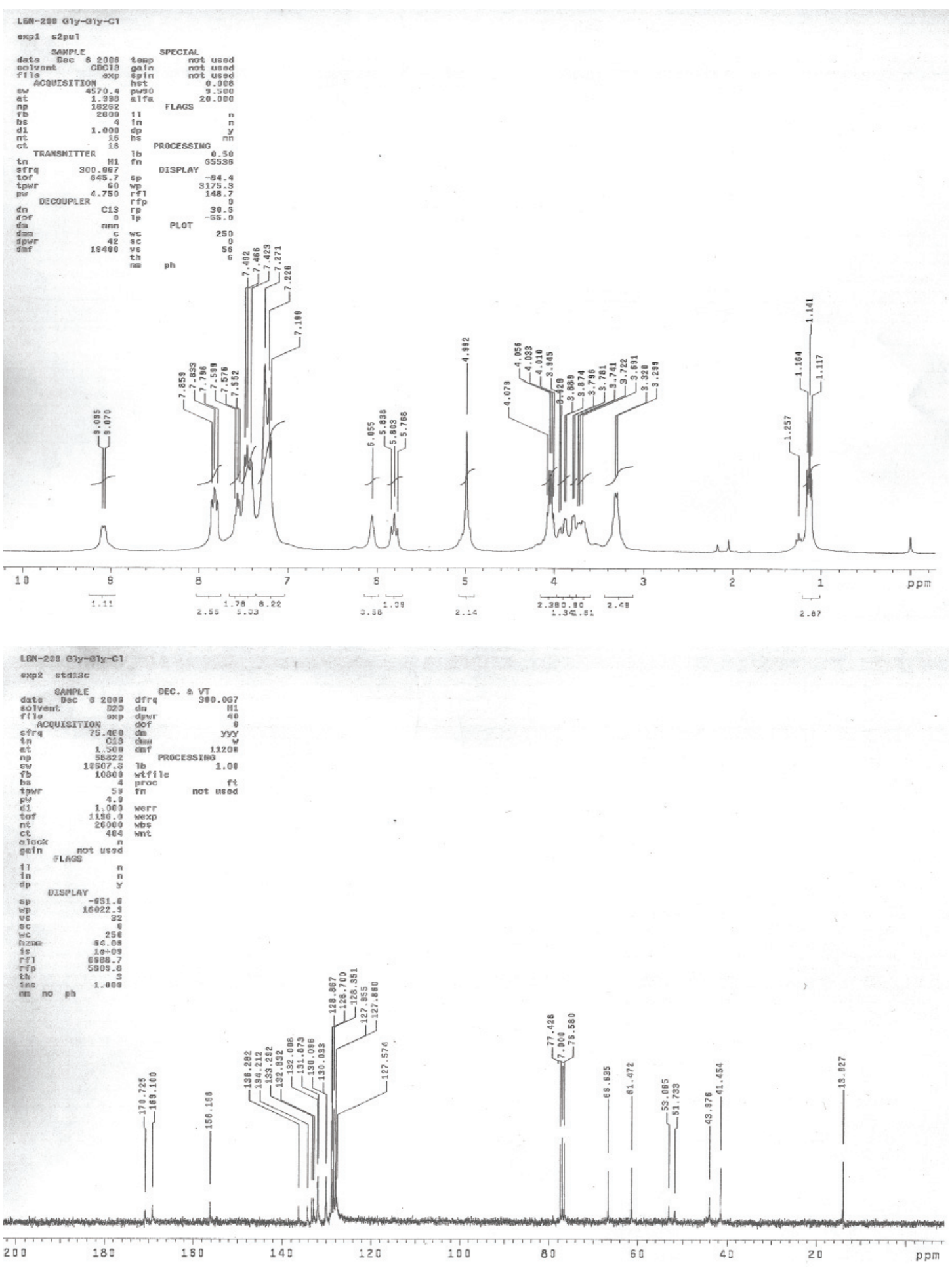


<smiles>CCOC(=O)CNP(=O)(c1ccccc1)C(NC(=O)CNC(=O)OCc1ccccc1)C(C)C</smiles>

Table 2 , entry $6\left(300 \mathrm{MHz}, \mathrm{CDCl}_{3}\right)$

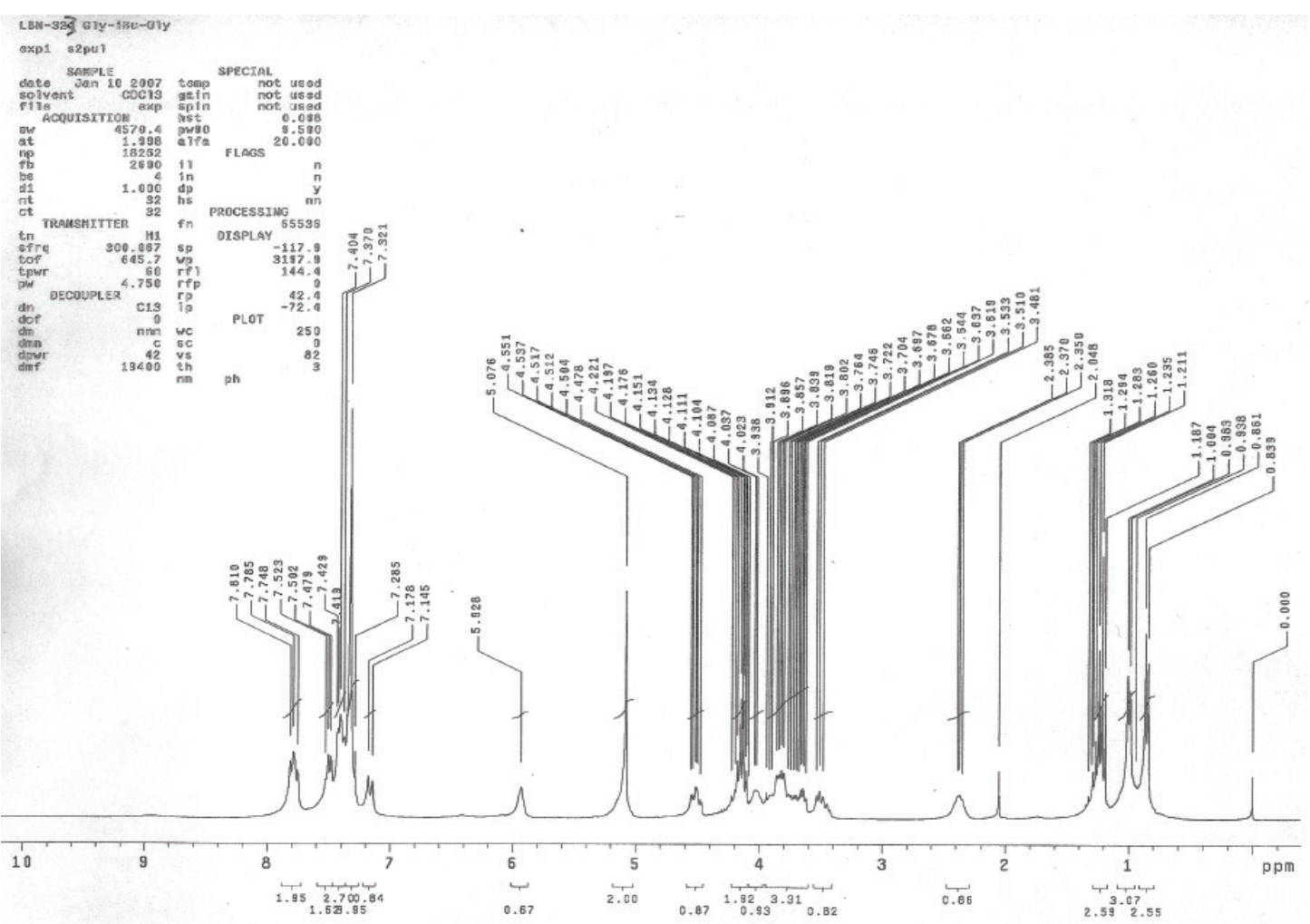

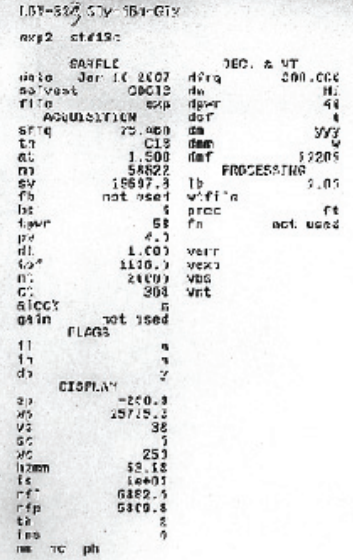

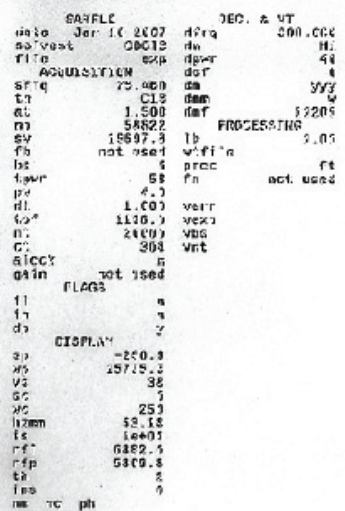

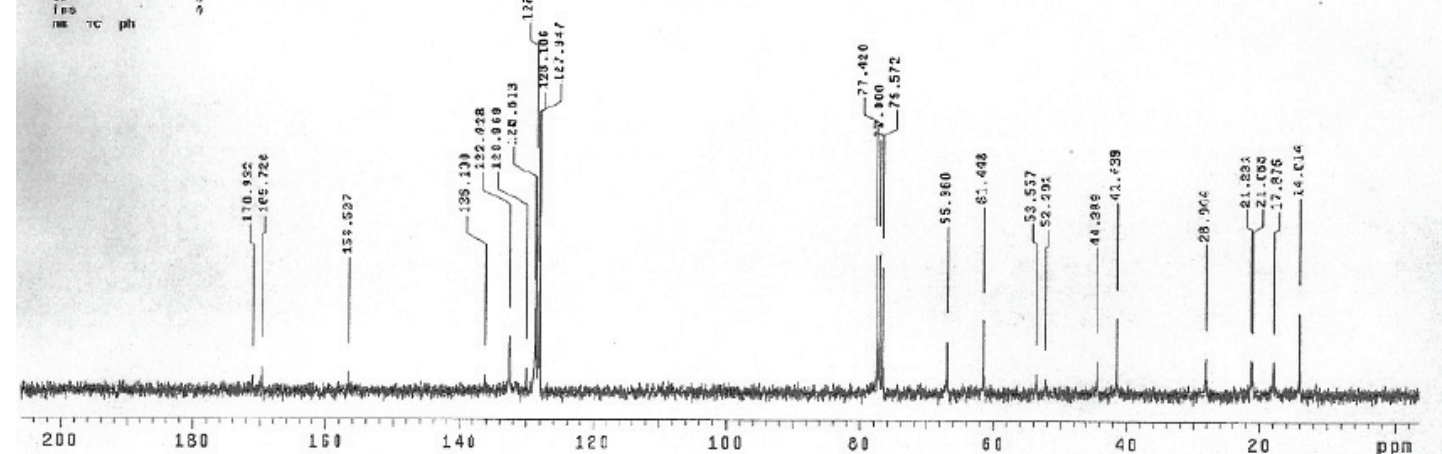


<smiles>CCCC(NC(=O)CNC(=O)OCc1ccccc1)[PH](O)(NCC(=O)OCC)c1ccccc1</smiles>

Table 2, entry $7\left(300 \mathrm{MHz}, \mathrm{CDCl}_{3}\right)$

LBN-895 GIy-nDu-819

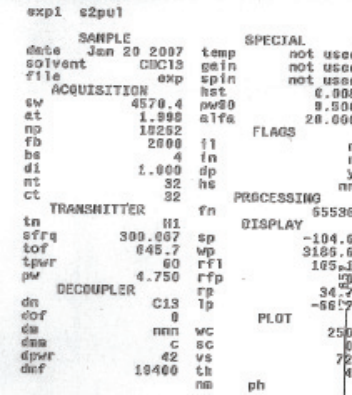

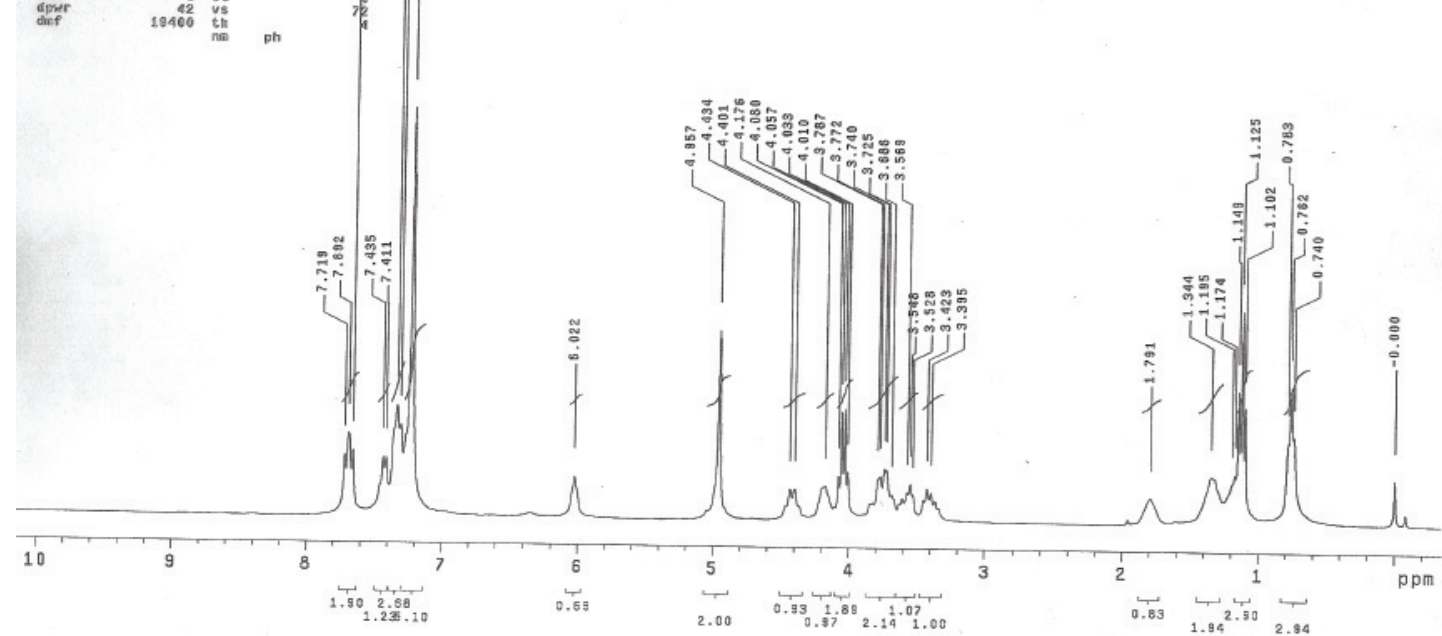

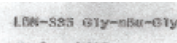

exp2 sta2sc

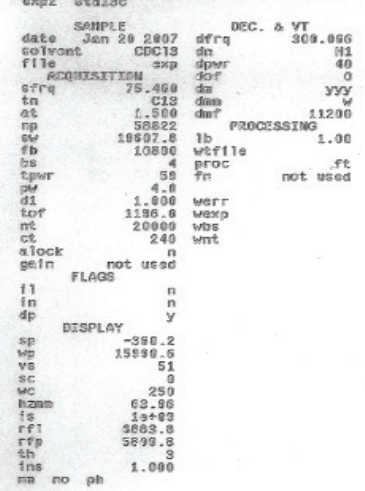

$1.9 e^{2.30}, 3.04$ 
<smiles>CCOC(=O)CNP(=O)(c1ccccc1)C(NC(=O)C(C)NC(=O)OCc1ccccc1)c1ccccc1</smiles>

Table 2 , entry $8\left(300 \mathrm{MHz}, \mathrm{CDCl}_{3}\right)$
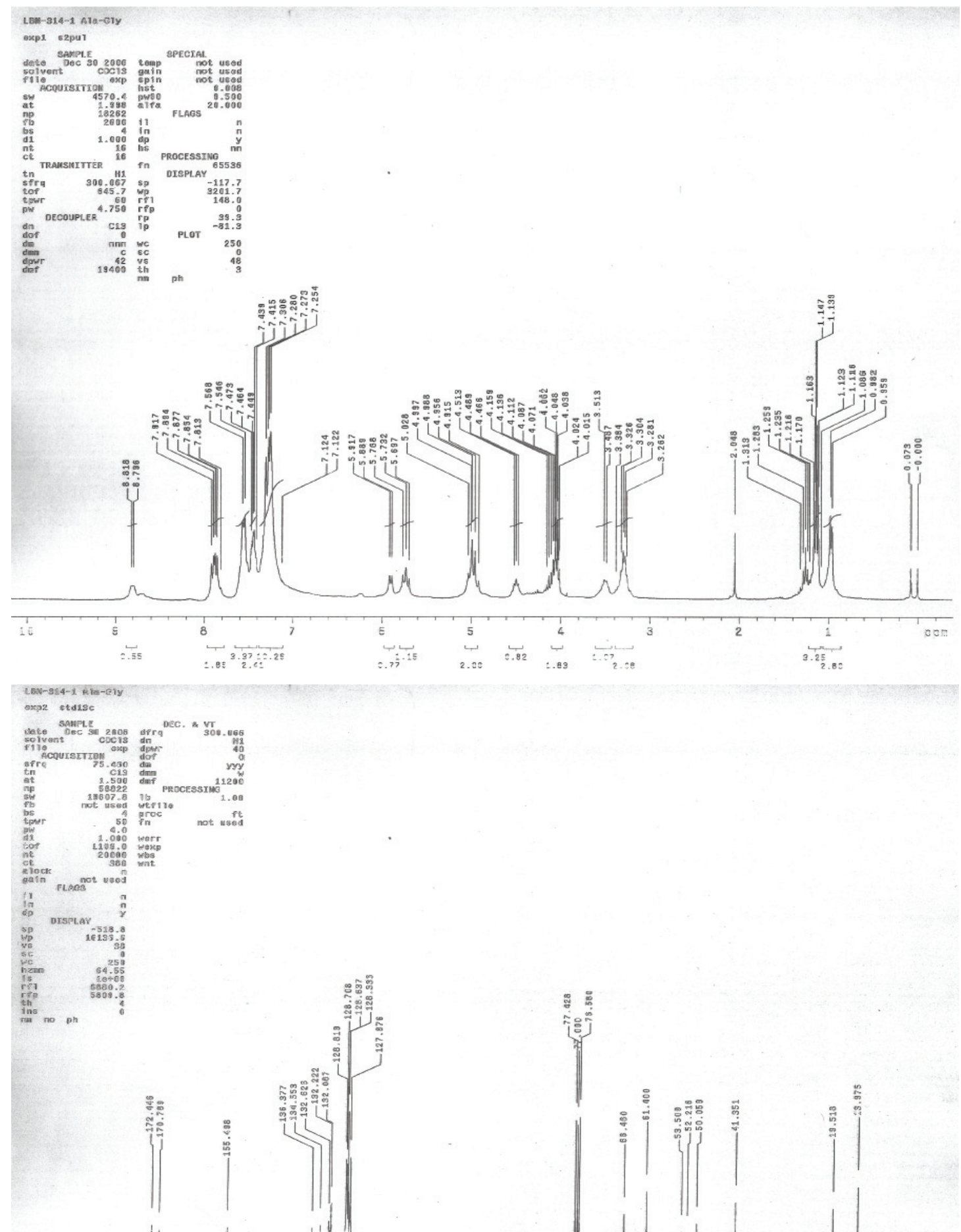
<smiles>CCOC(=O)CNP(=O)(c1ccccc1)C(NC(=O)CCNC(=O)OCc1ccccc1)c1ccccc1</smiles>

Table 2 , entry $9\left(300 \mathrm{MHz}, \mathrm{CDCl}_{3}\right)$
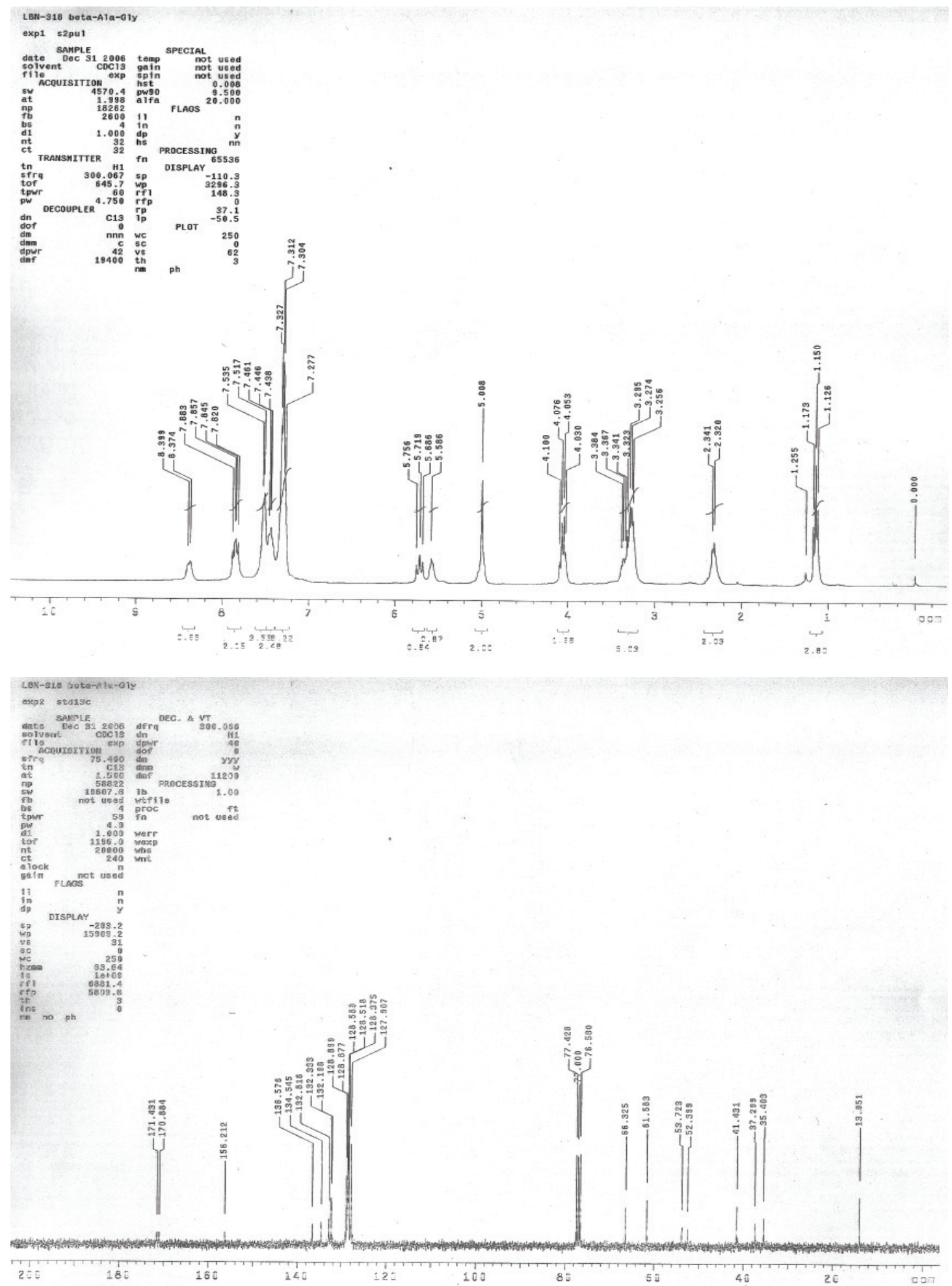

100 
<smiles>COC(=O)[C@H](Cc1ccccc1)NP(=O)(N[C@@H](Cc1ccccc1)P(=O)(O)c1ccccc1)[C@@H](Cc1ccccc1)NC(=O)CNC(=O)OCc1ccccc1</smiles>

Table 2, entry $10\left(300 \mathrm{MHz}, \mathrm{CDCl}_{3}\right)$

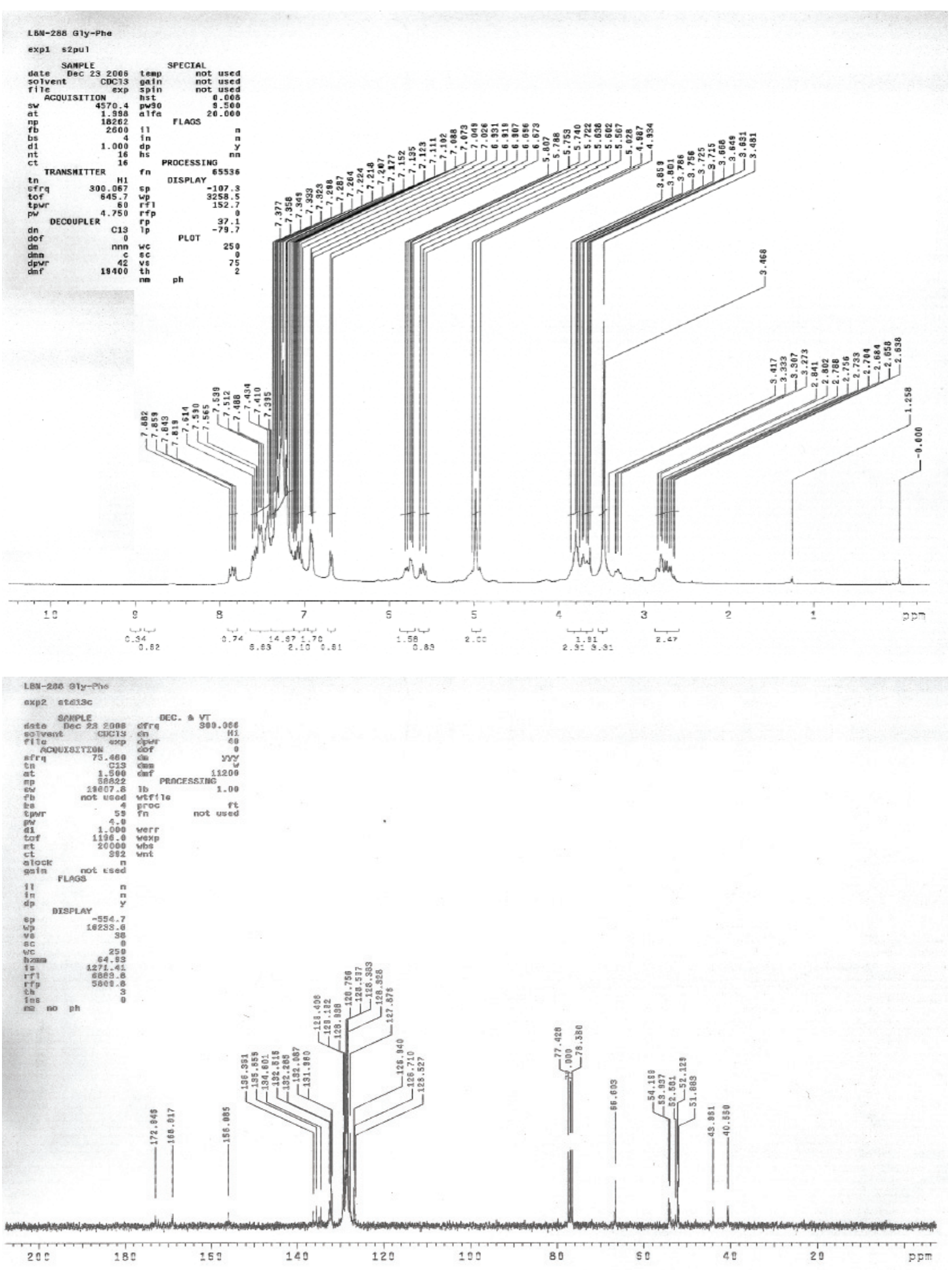


<smiles>CCOC(=O)CCNP(=O)(c1ccccc1)C(NC(=O)CNC(=O)OCc1ccccc1)c1ccccc1</smiles>

Table 2, entry $12\left(300 \mathrm{MHz}, \mathrm{CDCl}_{3}\right)$
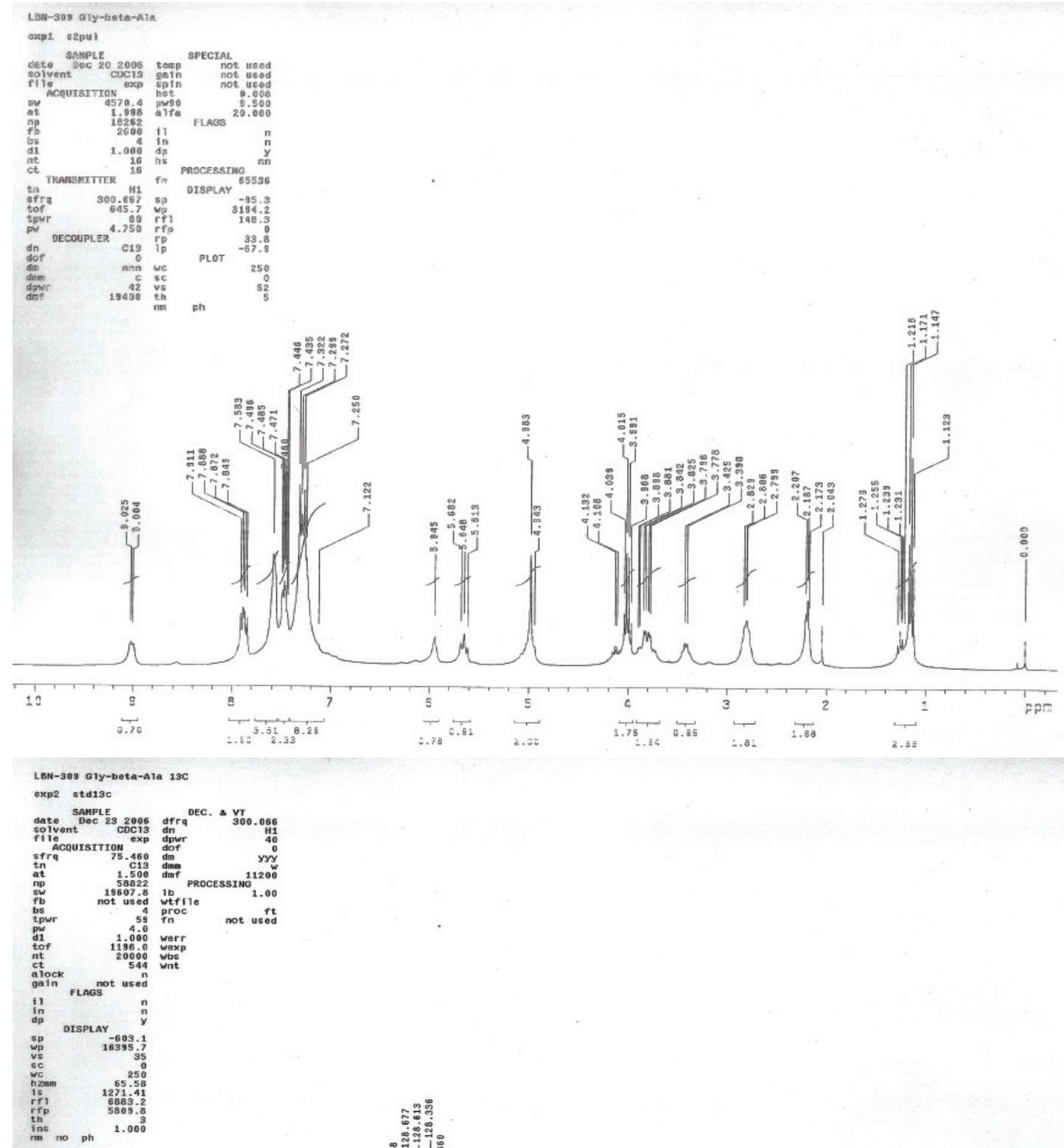
<smiles>CCOC(=O)CNC(=O)CNP(=O)(c1ccccc1)C(NC(=O)CNC(=O)OCc1ccccc1)c1ccccc1</smiles>

Table 2, entry $13\left(300 \mathrm{MHz}, \mathrm{CDCl}_{3}\right)$

LEN-931 61y-01yoly

exp1 s2pui

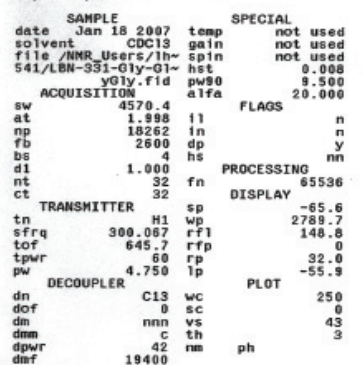

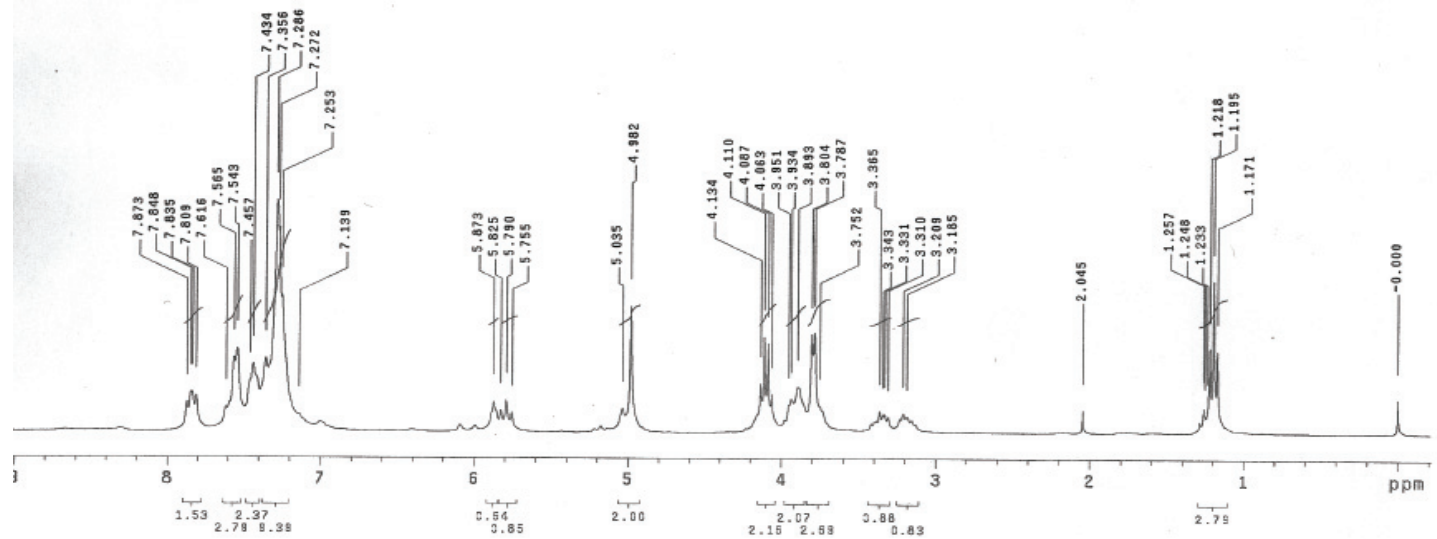

LBN-331 oly-61yGly

exp2 etd13c
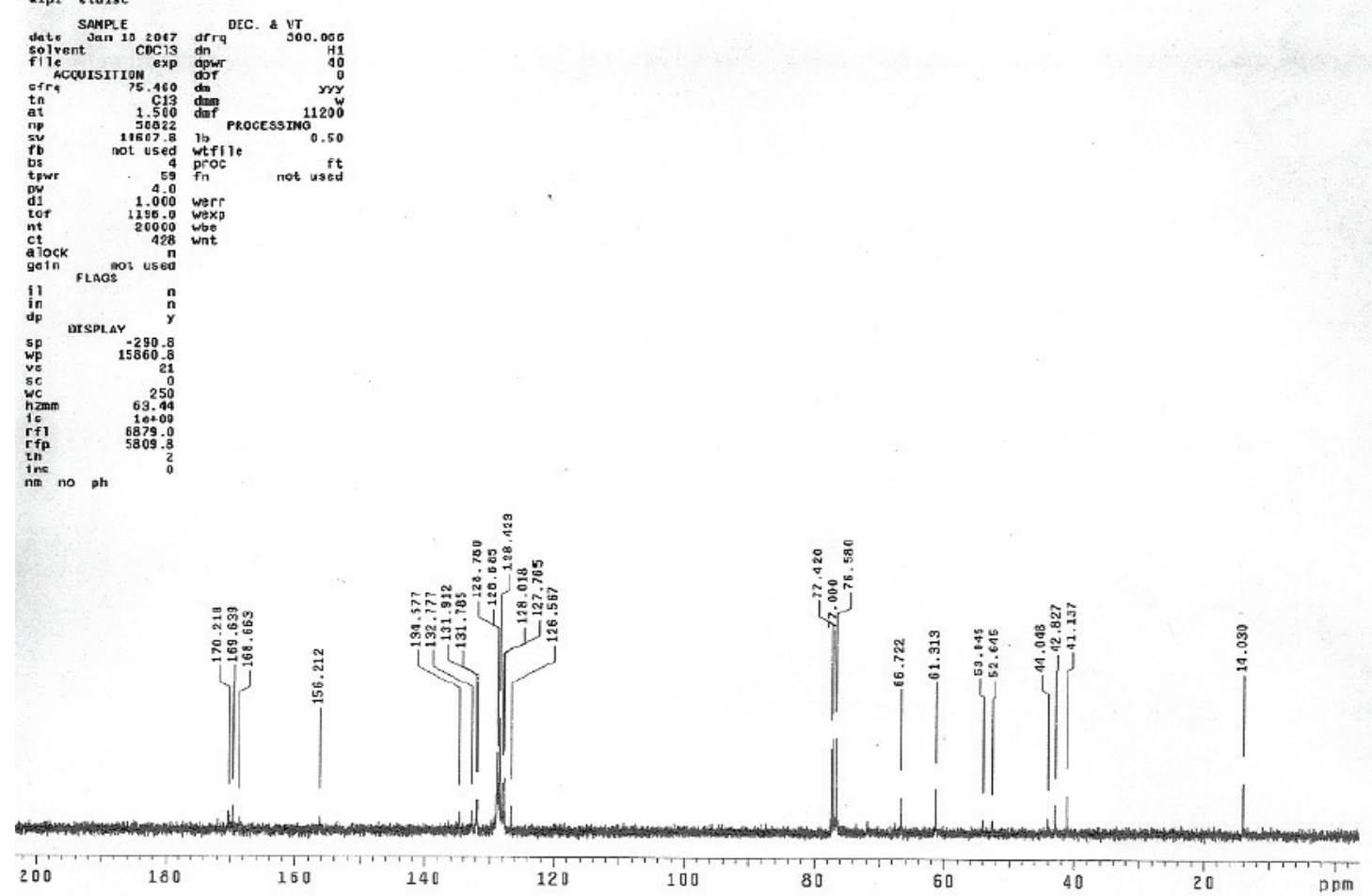
<smiles>CCOC(=O)CNP(=O)(c1ccccc1)C(NC(=O)CNC(=O)CNC(=O)OCc1ccccc1)c1ccccc1</smiles>

Table 2, entry $15\left(300 \mathrm{MHz}, \mathrm{CDCl}_{3}\right)$
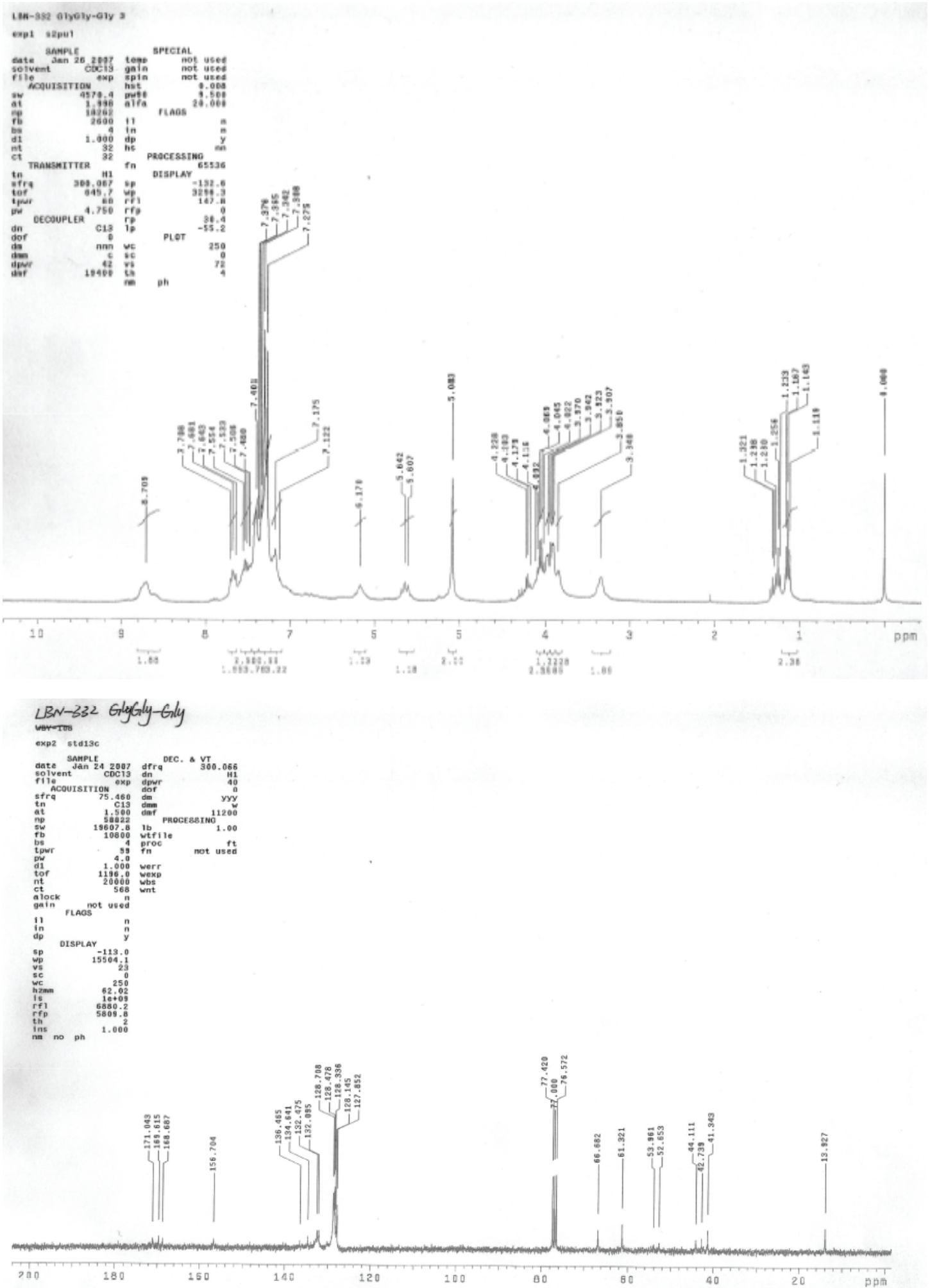